\title{
An Enthalpy Formulation for Thermocline with Encapsulated PCM Thermal Storage and Benchmark Solution using the Method of Characteristics
}

\author{
Eric Tumilowicz, Cho Lik Chan*, Peiwen Li, Ben Xu \\ Department of Aerospace and Mechanical Engineering \\ The University of Arizona \\ 1130 N. Mountain Ave., Tucson, AZ, 85721, USA \\ *Corresponding author. Tel.: 520-621-2503; Fax: 520-621-8191; \\ Email address: cholik@email.arizona.edu (Cho Lik Chan)
}

\begin{abstract}
An enthalpy-based model of thermocline operation applicable to both single phase and encapsulated phase change filler materials was developed. Numerical simulation of the model was created using MATLAB. The method of characteristics was applied in space and time, mapping fluid temperature and filler enthalpy to a numerical grid, and in the case of a melting filler, allowed accurate tracking of PCM filler phase state interfaces to fractional positions of the grid. Careful consideration of various possible heat transfer conditions along with placement of PCM filler phase state interfaces in the numerical grid allowed for great versatility and accuracy in model application. Input of fluid and filler properties, tank size, time of operation, and initial and boundary conditions to the program returned a full representation to any desired amount of charge/discharge processes or cycles. The paper covers mathematical formulation, certain intricacies of numerical implementation, model verification, and the beginnings of application to prove proper operation and generality.
\end{abstract}

Key words: Concentrated Solar Power (CSP), Latent Heat Storage System, Phase Change Material (PCM), Enthalpy Method, Method of Characteristics

1|P a g e

(C) 2014. This manuscript version is made available under the Elsevier user license http://www.elsevier.com/open-access/userlicense/1.0/ 


\section{Nomenclature}

\begin{tabular}{|c|c|c|c|}
\hline$c_{r}^{*}$ & Heat capacities ratio of PCM, solid to liquid & $\eta$ & Dimensionless enthalpy \\
\hline$d_{r}$ & Diameter of encapsules (m) & $\theta$ & Dimensionless temperature \\
\hline$H$ & Overall height of storage tank (m) & $\mu$ & Dynamic viscosity (kg/ms) \\
\hline$H_{C R}$ & Dimensionless heat capacity ratio & $v$ & Kinematic viscosity $\left(\mathrm{m}^{2} / \mathrm{s}\right)$ \\
\hline$h$ & heat transfer coefficient $\left(\mathrm{W} / \mathrm{m}^{2} . \mathrm{K}\right)$ & $\rho$ & Density $\left(\mathrm{kg} / \mathrm{m}^{3}\right)$ \\
\hline $\bar{h}$ & Enthalpy (J/kg) & $\tau_{r}$ & Dimensionless time scale \\
\hline$k$ & Thermal conductivity (W/m.K) & & \\
\hline $\operatorname{Pr}$ & Prandtl number & & \\
\hline$R$ & Radius of storage tank (m) & & \\
\hline $\operatorname{Re}$ & Reynolds number & Subscripts & \\
\hline$r$ & Radius of packed bed particle (m) & $f$ & Refers to HTF \\
\hline $\mathrm{S}$ & Surface area $\left(\mathrm{m}^{2}\right)$ & $H$ & Refers to the highest value of a variable \\
\hline$S_{r}$ & Surface area per length scale (m) & $o$ & Refers to the initial condition of a variable \\
\hline Stf & Stefan number & $L$ & Refers to the lowest value of a variable \\
\hline$T$ & Temperature $\left({ }^{\circ} \mathrm{C}\right)$ & $l$ & Refers to the liquidus interface \\
\hline$t$ & Time (s) & $r$ & Refers to solid filler material \\
\hline$U$ & Axial Velocity in storage tank (m/s) & ref & Refers to the reference value of a variable \\
\hline$V$ & Volume $\left(\mathrm{m}^{3}\right)$ & $r_{-} r e f$ & Refers to a filler reference value \\
\hline \multirow[t]{2}{*}{$Z$} & Axial tank location (m) & $r_{-}$melt & Refers to a filler melting point value \\
\hline & & $r_{-} s$ & Refers to the filler in a solid phase state \\
\hline \multicolumn{2}{|c|}{ Greek Symbols } & $r_{-} l$ & Refers to the filler in a liquid phase state \\
\hline$\varepsilon$ & Porosity of packed bed & $S$ & Refers to the solidus interface \\
\hline
\end{tabular}

\section{Introduction}

With the constant expansion of modern civilization on a global scale, the gap between energy supply and energy demand continues to grow. Such a reality has driven many to investigate energy sources alternate to the typical fossil fuel offerings, of which solar is a great contender. Concentrated Solar Power (CSP) plants harvest the sun's radiation and convert the concentrated heat to electric energy. However, the resulting output is converted immediately, meaning the daylight supply does not correspond with evening peak demands [1]. To counteract this shortcoming, a thermal storage mechanism is applied, allowing a gathering of energy throughout hours of high solar 
density, to be discharged and applied as necessary at a later time. Of these storage mechanisms, we turn our attention to the thermocline.

Contrary to older two tank designs, the thermocline employs natural thermal stratification to allow thermal energy storage with a single tank- an obvious savings in material costs alone. For a charge process, initially cold fluid in the tank is extracted through the bottom, drawing in solar heated high temperature fluid into the top of the tank. This high temperature fluid heats the bed of filler material as it passes through, storing the thermal energy in the tank. When the stored energy is desired, the discharge process pulls the high temperature fluid from the top of the tank, to be replaced with cold fluid at the bottom, together extracting the energy back from the filler. The discharged high temperature fluid is used to drive an external power cycle [2]. A schematic of this process is included for full understanding.

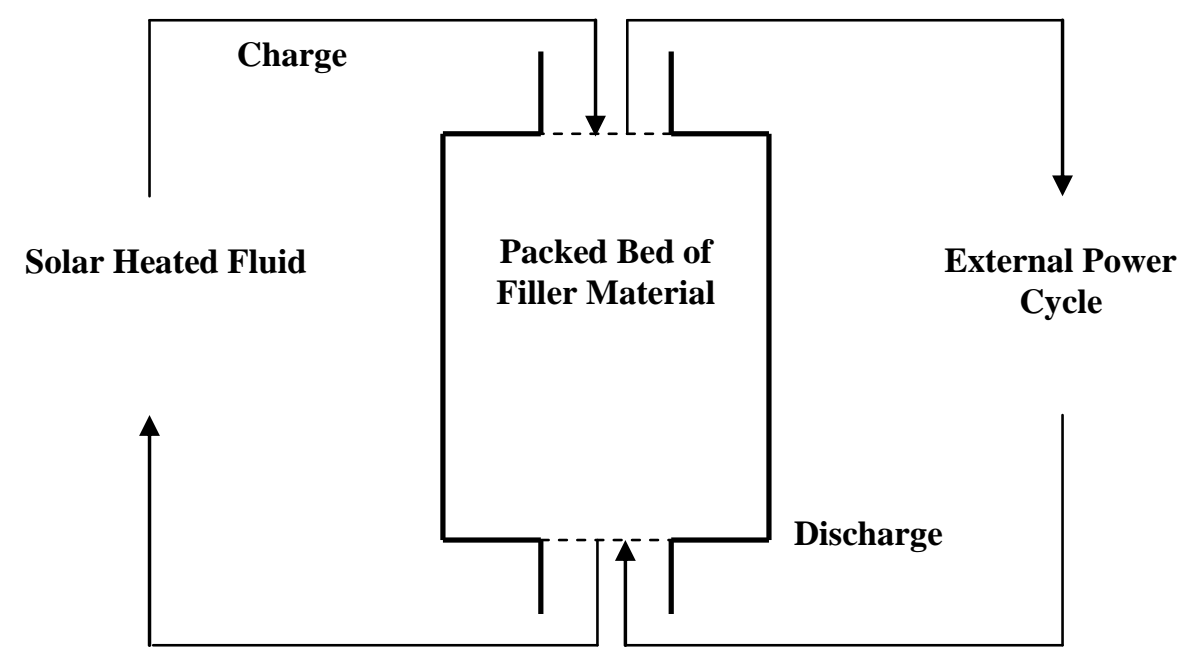

Fig. 1 General Thermocline Operation

In regards to thermocline filler material, the storage tank can operate on sensible heat, latent heat, or a combination of both. Based on properties alone, the use of a phase change material (PCM), combining both sensible and latent heat, allows a significantly higher energy storage density as compared to the use of sensible heat exclusively. Experimental studies of various tank filler materials confirm enhanced performance through use of a PCM, show resulting tank volume reduction by as much as a factor of 10, and suggest PCM fillers as fully viably alternatives for all thermal energy storage applications [3-6].

Efforts in thermal energy storage modeling go back as far as Schumann in 1929 [7], whose equations set the basis for representing fluid flow through a porous packed bed thermal storage tank. Following models [8, 9] expand consideration, where most recently, Van Lew applied the Method of Characteristics to produce a direct, fast, and accurate numerical solution to model thermocline interactions [10]. A model by Felix Regin and Solanki [11] considered a simple charge process of a tank with PCM filler for a parametric study of material properties. Following, a model by Wu et al. [12] applied an implicit finite difference method to solve the equations for the case with presence of PCM filler in the tank as a more general scenario, though results from the model featured numerous oddities and oscillations in temperature distribution profiles. To overcome the lower thermal conductivity of PCM 
material, Nithyanandam and Pitchumani $[13,14]$ introduced heat transfer augmentation using thermosyphons or heat pipe. Different configurations were investigated by using CFD. Optimal orientation and design parameters were obtained. Archibold et al. [15] focused their attention on the fluid flow and heat transfer of the PCM within the spherical encapsulate. Recirculating vortexes were found in the upper region and therefore more intense melting occurs in this region. On the other hand, Vyshak and Jilani [16] used a modified enthalpy method to investigate the melting times for rectangular, cylindrical, and cylindrical shell storage configurations. The melting time was the least for cylindrical shell storage. They also investigated the effects of inlet temperature of the heat transfer fluid. Nithyanandam et al. [18] developed a transient numerical model for a latent thermocline storage system with encapsulated PCM. Repeated changing and discharging cycles were simulated to investigate the dynamic response. They presented a procedure for designing a thermocline tank packed with PCM. They found that using smaller encapsulated PCM greatly reduces the tank size. Flueckiger and Garimella [19] developed a new finite-volume approach to simulate the mass and energy transport inside a latent heat thermocline tank at low computational cost. System-level model, incorporating the transport model, was then developed to evaluate the viability of using latent heat storage. They found that thermocline tanks filled with a single PCM is not effective. They proposed a cascaded filler structure composed of multiple PCMs of different melting temperatures. In the present paper we proposed a robust, comprehensive, and highly accurate model for thermal energy storage with an encapsulated PCM filler.

The current work followed suite after the success of Van Lew, with a much-needed expansion of analysis to an encapsulated PCM filler [10]. An enthalpy-based version of the Schumann equations was used to allow tracking of interactions throughout the thermocline processes- a change especially necessary in the latent region where PCM filler temperature remained constant. The new set of equations was non-dimensionalized for general application. With the resulting equations being of hyperbolic type, the method of characteristics was applied for a numerical solution. The process gave fluid temperature and PCM filler enthalpy according to the discretized grid in time and space. With the equations following a similar form of those Van Lew obtained, we too expected the method to produce a direct solution that is both highly accurate and efficient.

The addition of enthalpy to consideration required an equation of state to close the gap in unknowns for solution. For proper application of this equation in the governing thermocline interactions, PCM filler phase states had to be tracked closely. More importantly, to maintain accuracy as these PCM filler phase states changes throughout the space, a careful tracking of PCM filler phase state interfaces had to be implemented as well. This allowed proper application of the equations to all possible orientations and conditions of the PCM filler phase state interfaces in the numerical grid of characteristics. The method of characteristics made this possible, though the extent of generality and versatility hinged on the completeness of physical cases considered in its application.

\section{Assumptions and Governing Equations}

To obtain the governing equations for fluid and PCM filler interactions in the thermocline, we first made some necessary assumptions. The work assumed a strictly vertical fluid flow through the tank, along with a uniform fluid distribution in the radial direction. This reduced consideration into a single spatial dimension ' $z$ ', which followed the direction of fluid flow. In this regard, ' $z$ ' became a coordinate system that can be chosen, allowing identical 
application of the governing equations to both charge and discharge processes. Fluid thermo-physical properties were assumed invariant with temperature, and thus constant. A general representation of the to-be-modeled thermocline tank can be viewed below.

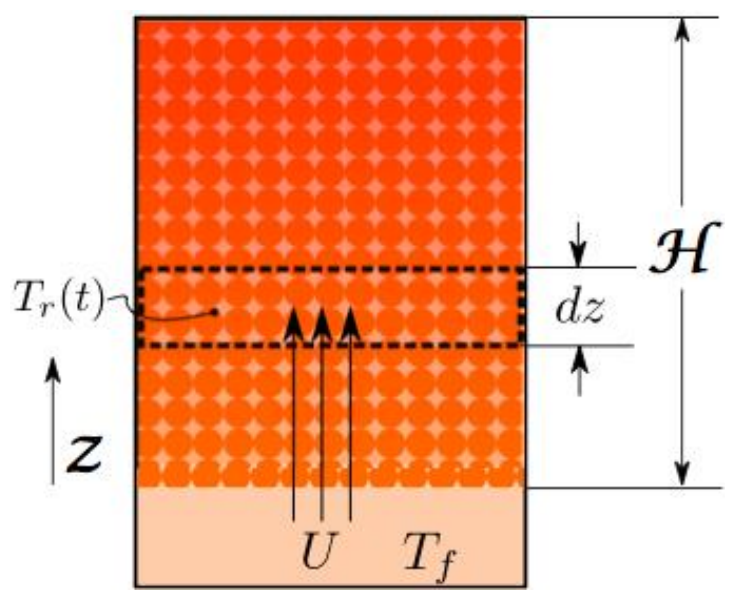

Fig. 2 General Thermocline for Modeling

Van Lew's analysis of the Peclet number of the tank [17] found it to be large, allowing heat conduction in the axial direction to be neglected for both PCM filler and fluid. We also assumed the storage tank was well insulated. In regards to the tank's filler material, we assumed it was a packed bed of spherical capsules of equal radius, each containing a fixed mass of PCM filler. For this, we required a lumped capacitance assumption, which allows any internal temperature gradients present in the encapsulated filler to be encompassed in its convection coefficient $h$ [20]. Van Lew previously confirmed the validity of this application via analysis of the Biot Number [17]. Though filler specific heat capacity was allowed to vary with changes in phase, the fixed mass and volume implemented by the encapsulation assumption implied a fixed density throughout all PCM filler states.

We continue to considerations for the combination of fluid and PCM filler in the storage tank. Within the tank, after insertion of the PCM filler, there was only a small amount of void volume left for the fluid. We defined this as the average void fraction

$$
\varepsilon=\frac{V_{f}}{V_{\tan k}}
$$

Experiments showed that the average void fraction followed as a function of the ratio of tank diameter to filler capsule diameter [21]. The capsules of PCM filler in constant radius in the packed bed allowed us to assume $\varepsilon$ as a constant, with which we could find the effective cross sectional area seen by the fluid and PCM filler, respectively.

$$
\begin{gathered}
A_{f}=\varepsilon * A_{\tan k}=\varepsilon * \pi R^{2} \\
A_{r}=(1-\varepsilon) * A_{\tan k}=(1-\varepsilon) * \pi R^{2}
\end{gathered}
$$

The filler particles were assumed to have point contact, though actual formation may vary. Thus, for convective heat transfer analysis, we defined the surface area per length of the tank as, $S_{r}$, as [22]: 


$$
S_{r}=\frac{f_{s} A_{r}}{r}
$$

where $f_{s}$ is the surface shape factor, which varies depending on the particle packing scheme. Previous analysis by Van Lew found this constant to vary between 2 and 3 [10]. Having assumed a body centered cubic packing scheme, the shape factor became

$$
f_{s}=\frac{6 \pi \sqrt{3}}{16} \approx 2.04
$$

Based on the Colburn factor relation [20], the heat transfer coefficient characterizing the convective interaction between the primary thermal storage material (porous media) and HTF can be found as follows [23]:

$$
\begin{gathered}
h=0.191 * \operatorname{Re}^{-0.278}\left(\frac{\dot{m}}{A_{f}}\right) c_{f} \operatorname{Pr}_{f}^{-2 / 3} \\
\operatorname{Re}=\frac{4 r_{\text {char }}}{v_{f} \rho_{f}}\left(\frac{\dot{m}}{A_{f}}\right) \\
\operatorname{Pr}_{f}=\frac{f \quad{ }_{f} c_{f}}{k}
\end{gathered}
$$

where the relation between the characteristic radius and average particle radius is

$$
r_{\text {char }}=\frac{\varepsilon \cdot r}{2(1-\varepsilon)}
$$

Values for fluid kinematic viscosity and fluid conductivity were obtained using the same numerical approximations Van Lew used in his work [10].

With assumptions covered, we applied an energy balance to both fluid and filler for the chosen differential control volume. For the fluid, necessary terms included the enthalpy of the flow in and out of the volume, energy exchange between the fluid and PCM filler material within the volume, and the internal energy change of the fluid over the instance in time. The fluid governing equation became:

$$
\frac{T_{f}}{t}+U \frac{T_{f}}{z}=\frac{h S_{r}\left(\begin{array}{ll}
T_{r} & T_{f}
\end{array}\right)}{{ }_{f} A_{f} c_{f}}
$$

For the PCM filler, we needed only consider the energy exchange between it and the fluid, and the change of internal energy of the PCM filler over the instance in time [24]. The PCM filler governing equation became

$$
\frac{\bar{h}_{r}}{t}=\frac{h S_{r}\left(T_{r} T_{f}\right)}{{ }_{r} A_{r}}
$$

The above equations still retain a PCM filler temperature term, for which we applied an equation of state to relate to the enthalpy per PCM filler phase state. 


$$
T_{r}= \begin{cases}\frac{\left(\bar{h}_{r}-\bar{h}_{r_{-} r e f}\right)}{c_{r_{-} s}}+T_{r_{-} r e f} & \bar{h}_{r}<\bar{h}_{r_{-} \text {melt }} \\ T_{r_{-} \text {melt }} & \text { for } \quad \bar{h}_{r_{-} \text {melt }} \leq \bar{h}_{r} \leq \bar{h}_{r_{-} \text {melt }}+L \\ \frac{\left(\bar{h}_{r}-\left(\bar{h}_{r_{-} \text {melt }}+L\right)\right)}{c_{r_{-} l}}+T_{r_{-} \text {melt }} & \bar{h}_{r_{-} \text {melt }}+L<\bar{h}_{r}\end{cases}
$$

For this enthalpy-based 1-D model, the lumped capacitance assumption is applied considering the fact that the size of encapsulated fillers is small that ensures a small Biot number. However, if the encapsulated filler material is large that gives a large Biot number, the internal thermal resistance becomes significant, and a modification to the lumped capacitance has to be considered. This can be done by introducing an effective convective heat transfer coefficient in Eqs. (10) and (11). Detailed derivation of the formula of the effective heat transfer coefficient has been provided by $\mathrm{Xu}$ et al. [25], and verification of the application of effective heat transfer coefficient has been demonstrated by Li et al. [26].

For generality, dimensionless analysis was applied to the governing equations by introducing the following variables. Temperatures $T_{H}$ and $T_{L}$ are characteristic to the working system, representing the highest temperature inlet fluid used for heating during the charge process, and the lowest temperature fluid used for cooling during the discharge process, respectively.

$$
\begin{gathered}
\theta_{f}=\frac{T_{f}-T_{L}}{T_{H}-T_{L}} \\
\theta_{r}=\frac{T_{r}-T_{L}}{T_{H}-T_{L}} \\
t^{*}=\frac{t}{H / U} \\
z^{*}=\frac{z}{H} \\
\eta_{r}=\frac{\bar{h}_{r}-\bar{h}_{r_{-} r e f}}{c_{r_{-} s}\left(T_{m e l t}-T_{L}\right)}
\end{gathered}
$$

The governing equations in dimensionless form followed as below. Note that subscripted 'melt' and ' $r \_r e f$ ' variables correspond to values plugged directly into their dimensionless variable (i.e. $\theta_{r}\left(T_{r}=T_{r_{-} m e l t}\right) \rightarrow \theta_{r_{-} m e l t}$ ).

$$
\frac{d \theta_{f}}{d t^{*}}+\frac{d \theta_{f}}{d z^{*}}=\frac{1}{\tau_{r}}\left(\theta_{r}-\theta_{f}\right)
$$




$$
\begin{aligned}
& \frac{d \eta_{r}}{d t^{*}}=\frac{-H_{C R}}{\tau_{r}} \frac{1}{\theta_{r_{-} \text {melt }}}\left(\theta_{r}-\theta_{f}\right)
\end{aligned}
$$

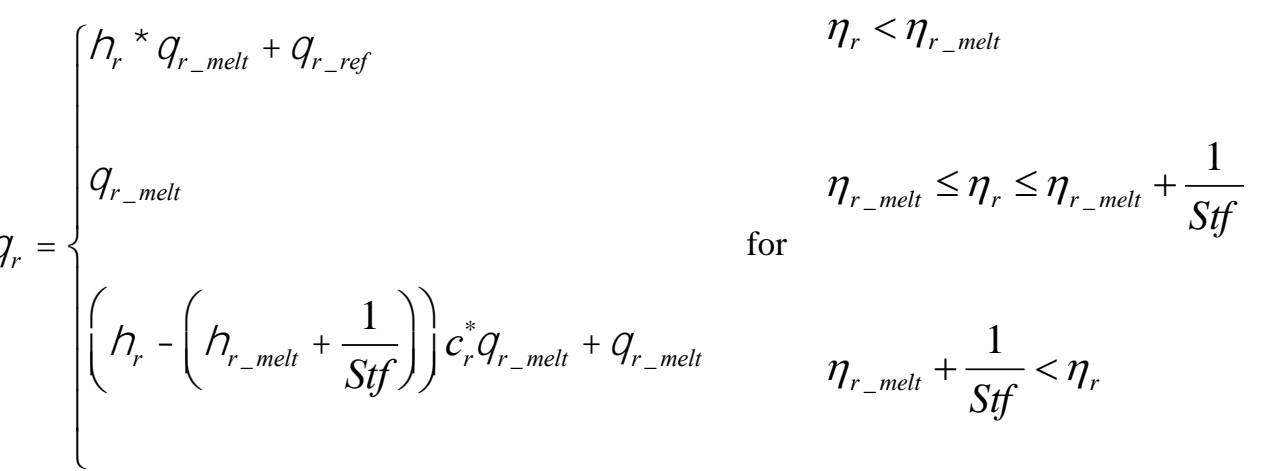

with dimensionless parameters defined as

$$
\begin{gathered}
\tau_{r}=\frac{U}{H} \frac{\rho_{f} c_{f} A_{f}}{h S_{r}} \\
H_{C R}=\frac{\rho_{f} c_{f} A_{f}}{\rho_{r} c_{r_{-} s} A_{r}} \\
S t f=\frac{c_{r_{-} s}\left(T_{r_{-} \text {melt }}-T_{L}\right)}{L} \\
c_{r}^{*}=\frac{c_{r_{-} s}}{c_{r_{-} l}}
\end{gathered}
$$

\section{Numerical Solution}

To solve the equations presented, the method of characteristics was applied. Using an equal step size in both time and space $\Delta t^{*}=\Delta z^{*}$, we chose a numerical grid featuring both diagonal characteristics $t^{*}=z^{*}$ and vertical characteristics $z^{*}=$ constant as shown in Fig. 3 below. Steps in time progressed for $j=1,2, \ldots \mathrm{N}$, while steps in space progressed for $i=1,2, \ldots \mathrm{M}$. 


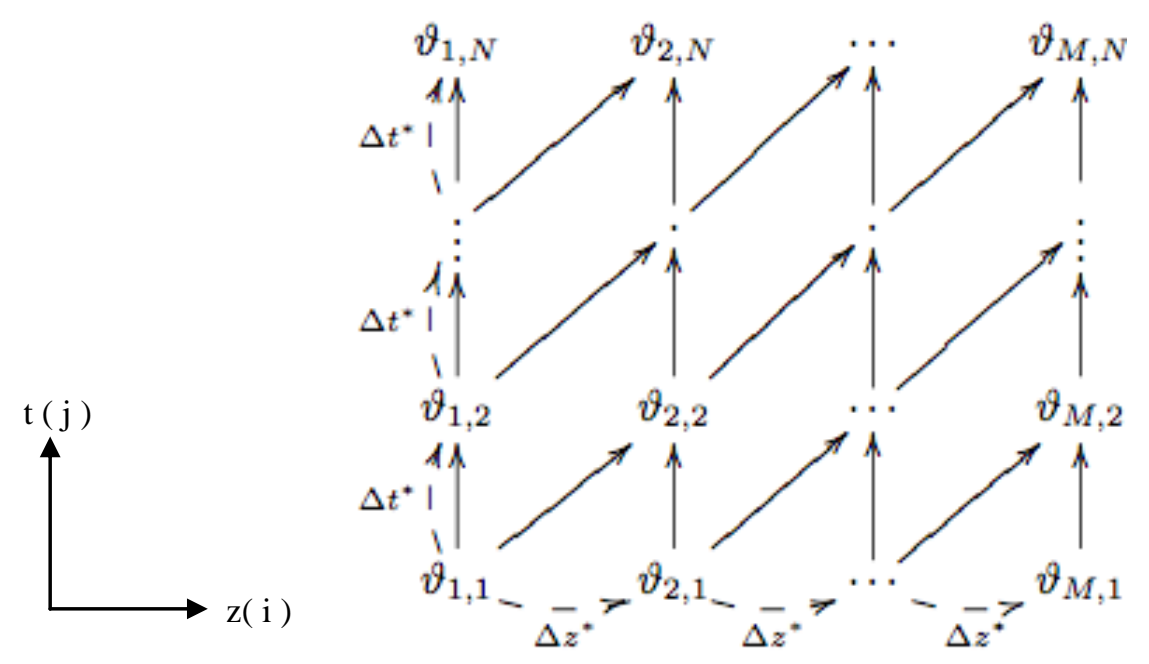

Fig. 3 Numerical Discretization Grid for the Method of Characteristics

Along the diagonal characteristic $t^{*}=z^{*}$, we recognized the substantial derivative in equation (18)

$$
\frac{D \theta_{f}}{D t^{*}}=\frac{1}{\tau_{r}}\left(\theta_{r}-\theta_{f}\right)
$$

Here, we first saw the advantage of applying the method of characteristics to this hyperbolic system. By choosing the diagonal characteristic, the fluid equation reduced from a partial differential equation to an ordinary differential equation along this curve. Separating and integrating along the characteristic, we obtained:

$$
\int d \theta_{f}=\int \frac{1}{\tau_{r}}\left(\theta_{r}-\theta_{f}\right) d t^{*}
$$

We continued with a similar process for the PCM filler energy balance. Equation (19) was solved along the characteristic $\mathrm{z}^{*}=$ constant. Again applying separation and integrating, we obtained

$$
\int d \eta_{r}=\int \frac{-H_{C R}}{\tau_{r}} \frac{1}{\theta_{r_{-} m e l t}}\left(\theta_{r}-\theta_{f}\right) d t^{*}
$$

Clearly, the result represented a system of ordinary differential equations along their corresponding characteristics. Referring back to the grid in Fig. 3, we saw the two characteristics intersect as they progress in time and space. We exploited this in our manner of solution throughout the nodal grid. The hyperbolic nature of the original equations passes information from node to node in a wave-like fashion. As an example, we chose two neighboring spatial nodes at time $j=1,(1,1)$ and $(2,1)$, which will serve as the starting points for information propagation through their corresponding characteristics. After the passing of one time step to $j=2$, the meeting point of the two characteristics, $(2,2)$, will have received information from the two starting nodes. To represent this mathematically, we applied numerical integration to the equations. Along the diagonal characteristic, equation (26) became 


$$
\int_{(1,1)}^{(2,2)} d \theta_{f}=\int_{(1,1)}^{(2,2)} \frac{1}{\tau_{r}}\left(\theta_{r}-\theta_{f}\right) d t^{*}
$$

Applying the trapezoidal rule for integration of the right hand side [4], the solution to equation (28) became

$$
\theta_{2,2}-\theta_{1,1}=\frac{\Delta t^{*}}{\tau_{r}}\left(\frac{\theta_{r_{2,2}}+\theta_{r_{1,1}}}{2}-\frac{\theta_{f_{2,2}}+\theta_{f_{1,1}}}{2}\right)
$$

Repeating the process for equation (27), we obtained

$$
\int_{(2,1)}^{(2,2)} d \eta_{r}=\int_{(2,1)}^{(2,2)} \frac{-H_{C R}}{\tau_{r}} \frac{1}{\theta_{r_{-} \text {melt }}}\left(\theta_{r}-\theta_{f}\right) d t^{*}
$$

Once again implementing the trapezoidal rule for integration of the right hand side [25], the integral solution became:

$$
\eta_{2,2}-\eta_{2,1}=\frac{-\Delta t^{*} H_{C R}}{\tau_{r} \theta_{r_{-} \text {melt }}}\left(\frac{\theta_{r_{2,2}}+\theta_{r_{2,1}}}{2}-\frac{\theta_{f_{2,2}}+\theta_{f_{2,1}}}{2}\right)
$$

Using the equation of state (20) to transform the unknown PCM filler temperature value at node $(2,2)$ to enthalpy based on local PCM filler phase state left the system of two equations (29) and (31) to solve for the two unknowns, $\theta_{f_{2,2}}$ and $\eta_{r_{2,2}}$. With these values obtained, we stepped once in space to the new pair of neighboring nodes, $(2,1)$ and $(3,1)$, and used them identically to obtain values at node $(3,2)$. This was repeated until all values have been found at $j=2$. We then fully repeated the spatial sweep at $j=2$ to obtain all new values at $j=3$.

Thus, with a boundary condition provided at the inlet $i=1$, along with an initial condition at time $j=1$ in the storage tank, solutions could be swept through space, stepped in time, and repeated, until the entire grid was fully calculated. Application of the trapezoidal rule for numerical integration implies accuracy of order $O\left(\Delta t^{* 2}\right)$ [27]. The above was a mere example calculation outlining the numerical process applied. With PCM filler phase state changes added, numerous technicalities had to be considered throughout the full application. While use of the state equation and solution of the system of equations followed simply in regions of continuous phase state, PCM filler phase state interfaces and their travel throughout the grid of characteristics created a plethora of more complex calculations. The necessary numerical considerations were most generally divided into calculations of type 'boundary' and 'spatial'. Within these, we covered all cases of heating and cooling between different types of PCM filler phase states, PCM filler phase state interface presence/positioning, and the possibilities of over-heating and under-cooling. These more extreme cases were a necessary consideration with the application of the method of characteristics. In the chosen numerical grid, information could only propagate at the characteristic speed. With extreme heating or cooling conditions, an interface could be found traveling faster than the maximum allowed $45^{\circ}$ (the diagonal characteristic), at which point its position had to be suppressed to the maximum, and the additional energy transfer was accumulated in the resulting PCM filler enthalpy at the phase state interface. 
At the boundary $(i=1)$, only the vertical characteristic was used for calculation, and known fluid values allowed direct calculation at this point in space for all instances in time. Analysis of PCM filler enthalpy results revealed any resulting phase state changes in the filler. Overall, the model included a total of 11 considerations for grid calculations of this type: (1) continuous solid phase state, (2) continuous melting phase state, (3) continuous liquid phase state, (4) solidus phase state interface onset with heating, (5) solidus phase state interface onset with cooling, (6) liquidus phase state interface onset with heating, (7) liquidus phase state interface onset with cooling, (8) solidus phase state interface onset with over-heating, (9) solidus phase state interface onset with under-cooling, (10) liquidus phase state interface onset with over-heating, (11), liquidus phase state interface onset with undercooling.

To handle the discretized grids in the tank space, we worked with the system of equations resulting from the intersection of the two characteristics. Specific calculation depended on the phase state(s) present, and the phase state interface placement amongst the corresponding characteristic lines. To better detail the process, we considered a case where phase state interface travel during an instance in time leaves it within the same block in the discretized grid. With all values at the current time known, we first shifted the solution characteristics to solve for the phase state interface position at the new time, $\beta_{\text {New }}$, which represented a fractional positioning of the phase state interface in the grid $0<\beta<1$. The corresponding general solution space in the discretized grid can be viewed below.

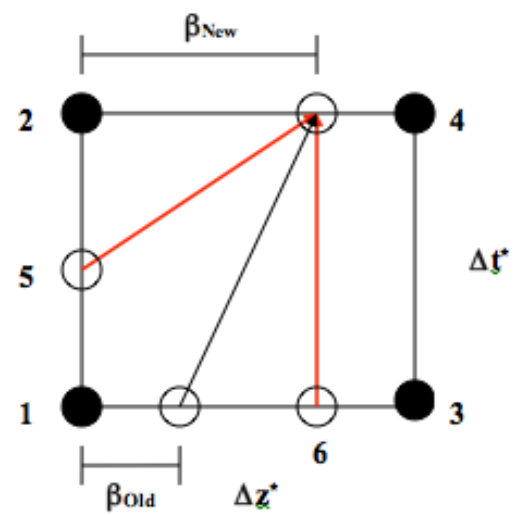

Fig. 4 Solution Grid for First Step of Standard Spatial Interface Travel

The characteristics were discretized as before, with integration of the fluid equation from point ' 5 ' to the interface location $\beta_{\text {New }}$, and of the PCM filler enthalpy equation from point ' 6 ' to the interface location $\beta_{\text {New }}$. Application of linear interpolation (using the nearest known points) for intermediate terms ' 5 ' and ' 6 ', followed by elimination of the unknown fluid temperature at $\beta_{\mathrm{New}}$ from the system, resulted in a second-degree polynomial to solve for $\beta_{\text {New }}$. From the previously mentioned definition, $0<\beta<1$, selection of the proper root followed simply. With the position at the new time found, the corresponding fluid value was obtained from the same characteristic equations. 
With travel of the phase state interface now defined within the grid, we continued to define necessary values at the upper right point in the spatial sweep. Due to the presence of a phase state interface, the solution characteristic must have been divided accordingly. The general procedure is depicted below.

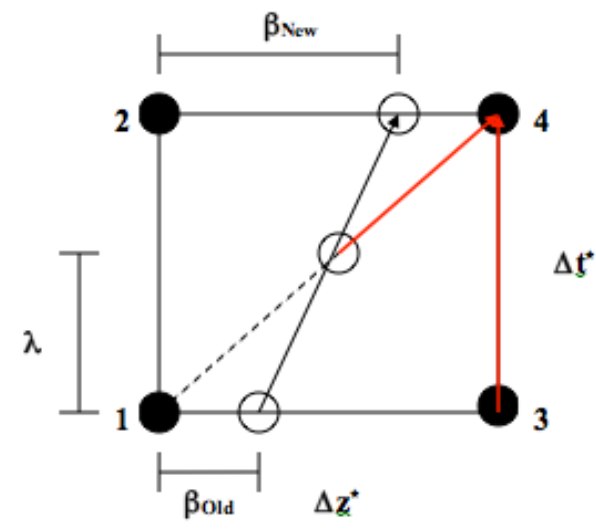

Fig. 5 Solution Grid for Second Step of Standard Spatial Interface Travel

Linear interpolation was applied along the interface for values at its intersection with the diagonal characteristic. Finally, the system of equations defined by the two above characteristics was solved for the fluid and PCM filler temperatures at point ' 4 '.

Overall, the model included 19 considerations for grid calculations of 'spatial' type: (1) continuous solid phase state, (2) continuous melting phase state, (3) continuous liquid phase state, (4) solidus phase state interface within grid with heating, (5) solidus phase state interface within grid with cooling, (6) liquidus phase state interface within grid with heating, (7) liquidus phase state interface within grid with cooling, (8) solidus phase state interface crossing vertical characteristic with heating, (9) solidus phase state interface crossing vertical characteristic with cooling, (10) liquidus phase state interface crossing vertical characteristic with heating, (11) liquidus phase state interface crossing vertical characteristic with cooling, (12) solidus phase state interface leaving spatial domain with heating, (13) solidus phase state interface leaving the spatial domain with cooling, (14) liquidus phase state interface leaving the spatial domain with heating, (15) liquidus phase state interface leaving the spatial domain with cooling, (16) solidus phase state interface crossing vertical characteristic with over-heating, (17) solidus phase state interface crossing vertical characteristic with under-cooling, (18) liquidus phase state interface crossing vertical characteristic with over-heating, (19), liquidus phase state interface crossing vertical characteristic with under-cooling.

\section{Verification}

Reviewing the final governing equations of interactions in the storage tank, educated choices in spatial positioning and PCM filler phase state allowed for analytic solutions to this otherwise complicated pair of equations. Exploiting these simplifications, and having access to the previous model produced by Van Lew [9], we were able to obtain additional confidence in the new model. 
The first step for verification featured a test up the tank inlet boundary. Here, fluid temperature was known as the inlet condition, which we assumed to be constant for simplicity. Also assuming solid PCM filler only with reference temperature being equal to the low temperature in the system, equation (19) reduced to a simple ordinary differential equation, whose solution followed simply.

$$
\begin{aligned}
& \frac{d \theta_{r}}{d t^{*}}+\frac{H_{C R}}{\tau_{r}} \theta_{r}=\frac{H_{C R}}{\tau_{r}} \theta_{f_{\text {inlet }}} \\
& \theta_{r}=\theta_{f_{\text {inlet }}}+\left(\theta_{r_{O}}-\theta_{f_{\text {inlet }}}\right) e^{\frac{-H_{C R} \tau_{r}^{*}}{\tau^{*}}}
\end{aligned}
$$

Thus, we obtained an analytical result for the solid PCM filler temperature profile at the boundary over time. We ran a representative case of the model, using a step size of $\Delta \mathrm{t}^{*}=\Delta \mathrm{z}^{*}=0.001$, and running to a dimensionless time of 4.0. The melting temperature was set above the high temperature to ensure the PCM filler remained in a solid phase state throughout the entire calculation. The remainder of the key parameters can be viewed below.

$$
\begin{aligned}
& H_{C R}=0.5785 \\
& \tau_{r}=0.1117 \\
& \theta_{f_{\text {inlet }}}=1 \\
& \theta_{r_{O}}=0
\end{aligned}
$$

The results from the model, as well as the results from the analytic solution in equation (33), can be viewed in Fig. 6 below. Clearly, the results are one and the same. Comparing at each point, the largest deviation in the 4000 points is a mere $0.000223 \%$, which can comfortably be allotted to numerical round off and truncation errors in the model. 


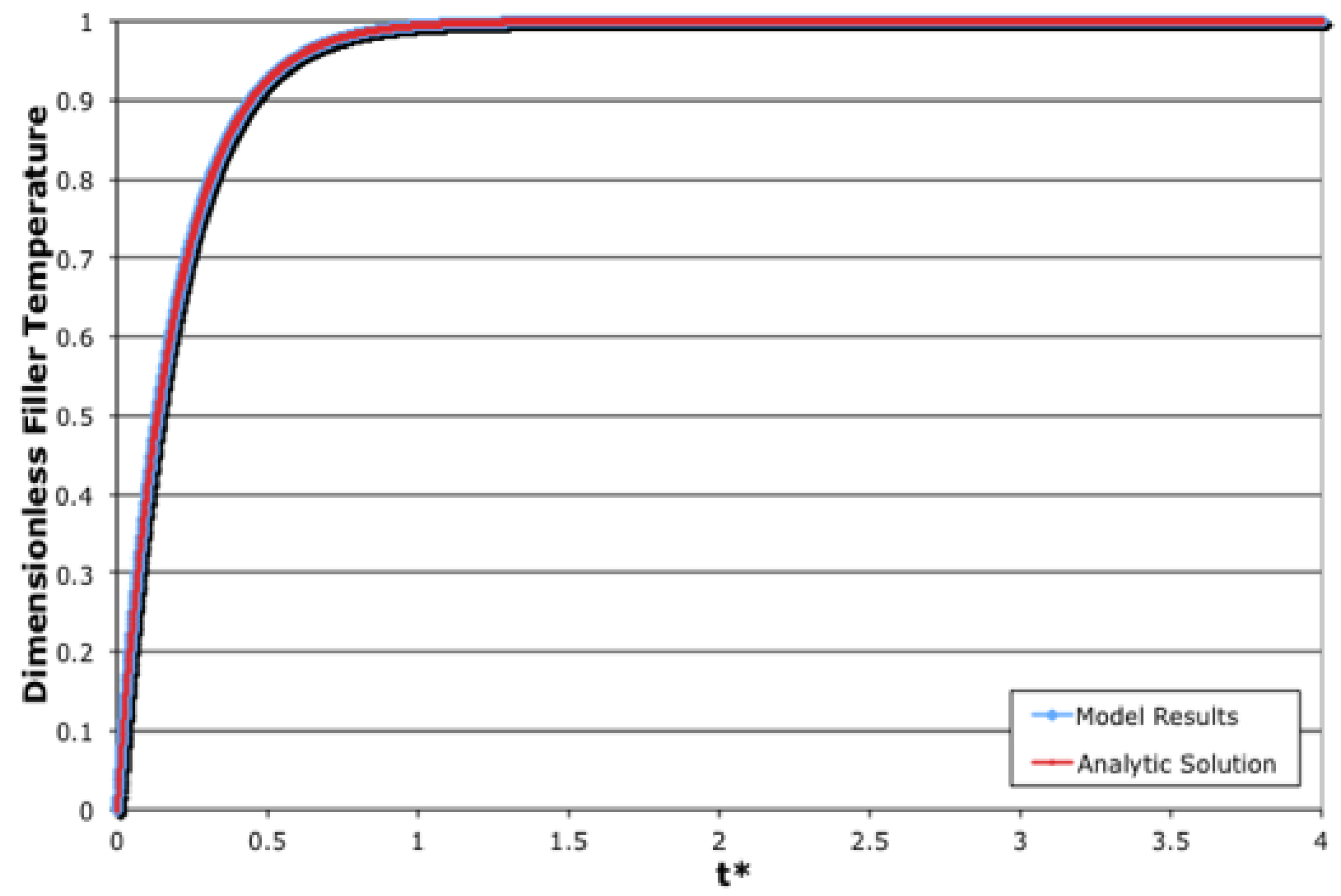

Fig. 6 Boundary Filler Temperature as a Function of Time

A second test for verification of the model was performed for a melting PCM filler. In this phase state, the PCM filler maintains a constant temperature $\theta_{r_{-} \text {melt }}$, which was exploited to allow analytic solutions of the fluid temperature and PCM filler enthalpy gradient equations. Assuming a constant inlet fluid condition and constant initial PCM filler condition, we transformed the fluid equation into the Laplace domain, where it became an ordinary differential equation that could be easily solved. We further assumed $t^{*}>z^{*}$, which simplified the result. Applying this to the PCM filler equation allowed simple solution via integration, the final analytic solutions for fluid temperature and PCM filler enthalpy with the above assumptions followed as

$$
\begin{gathered}
\theta_{f}\left(z^{*}\right)=\theta_{r_{-} \text {melt }}+\left(\theta_{f_{-} \text {inlet }}-\theta_{r_{-} \text {melt }}\right) e^{-\frac{z^{*}}{\tau_{r}}} \\
\eta_{r}\left(t^{*}, z^{*}\right)=\eta_{r_{-} o}+\frac{H_{C R}}{\tau_{r} \theta_{r_{-} \text {melt }}}\left(\theta_{f_{-} \text {inlet }}-\theta_{r_{-} \text {melt }}\right) e^{-\frac{z^{*}}{\tau_{r}}}\left(t^{*}-z^{*}\right)
\end{gathered}
$$

With these solutions obtained, we could run the model for a representative case, and compare results for verification. As this equation is valid for melting PCM filler only, we used a case with the initial condition of the tank featuring PCM filler at the onset of melting, and then heat with a high input temperature. Parameters used can be viewed below. 


$$
\begin{aligned}
& H_{C R}=0.5785 \\
& \tau_{r}=0.1117 \\
& \theta_{f_{-} \text {inlet }}=1 \\
& \theta_{r_{-} o}=\theta_{f_{-} o}=\theta_{r_{-} \text {melt }}=0.5
\end{aligned}
$$

We again used step sizes $\Delta t^{*}=\Delta z^{*}=0.001$, and applied the model to a final dimensionless time of 1.5. This short time frame was necessary to maintain only melting PCM filler in the tank. Shortly after, these conditions send the PCM filler into the liquid phase state, which had to be avoided for valid comparison to the derived equations. Results were compared across the entire spatial domain at the final instance in time, as this region is fully within the condition $t^{*}>z^{*}$, as applied for the analytic solution above. A comparison of fluid temperature and PCM filler enthalpy profiles can be seen in Fig. 7 and Fig. 8, respectively.

Clearly, the two results coincide well. For fluid temperature, amongst the 1000 points, the highest relative error is found to be $0.000186 \%$. For PCM filler enthalpy, the largest relative error is $0.0295 \%$. Though still a small percentage, this larger result reveals a residual error inherent to the method of characteristics and numerical integration. Due to the inlet fluid condition only being able to progress at the diagonal characteristic speed, the fluid temperature profile retains a discontinuity between the initial condition and portion affected by the inlet at any time before $t^{*}=1$. When work along a vertical characteristic for PCM filler enthalpy featured this discontinuity, the trapezoidal rule is not able resolve values as accurately for the governing equations, causing additional error as compared to the analytic solution. As the inlet fluid condition traverses through space, the discontinuity decreases, which in turn lower the resulting error, eventually to the same order of magnitude seen before. As calculations continued in time, any errors introduced in space are further decreased. Though resulting errors in the profiles become quite small at the end of run, it is important to understand this numerical intricacy inherent to the method. 


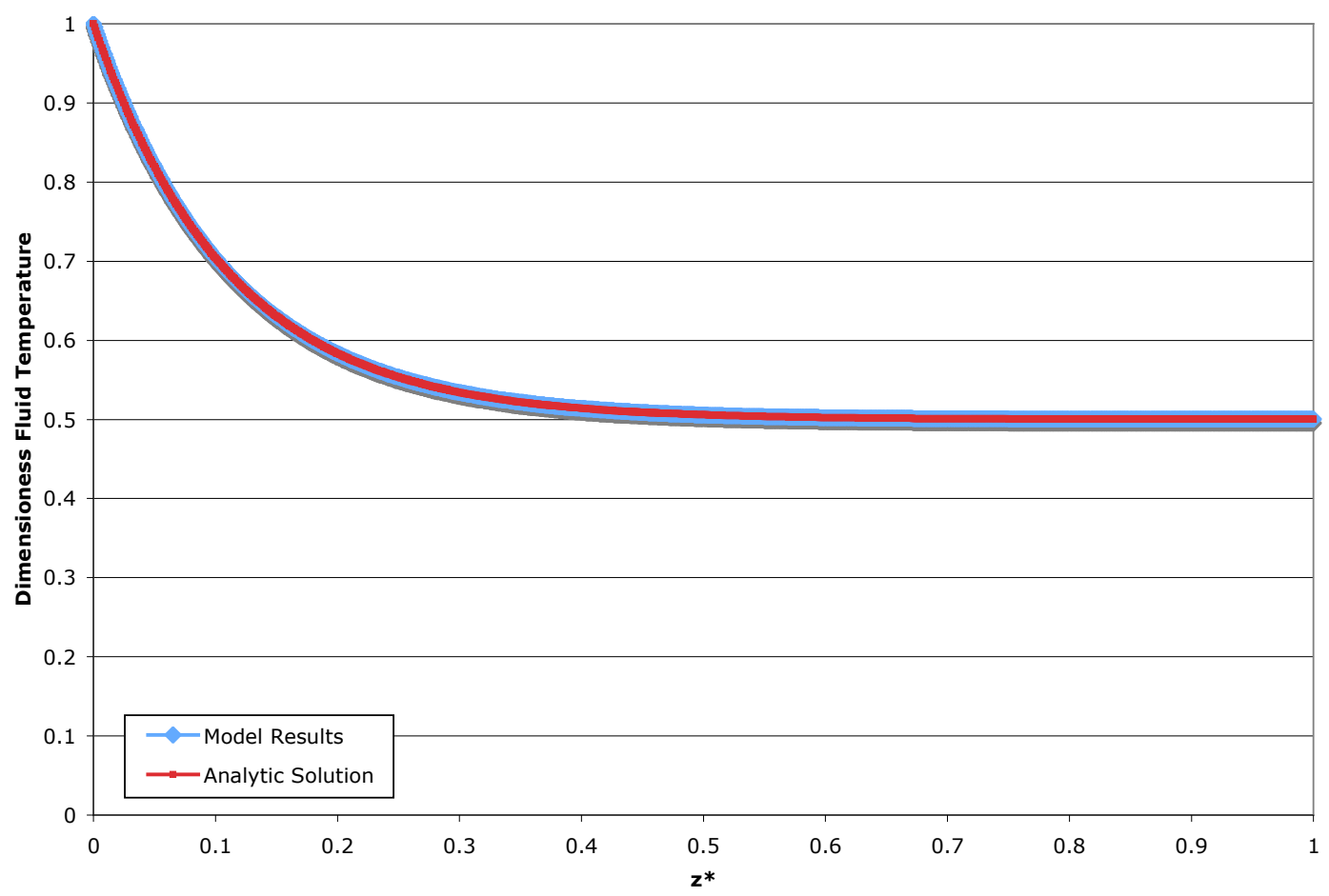

Fig. 7 Model and Analytic Solution Comparison of Fluid Temperature in Filler Melting Region

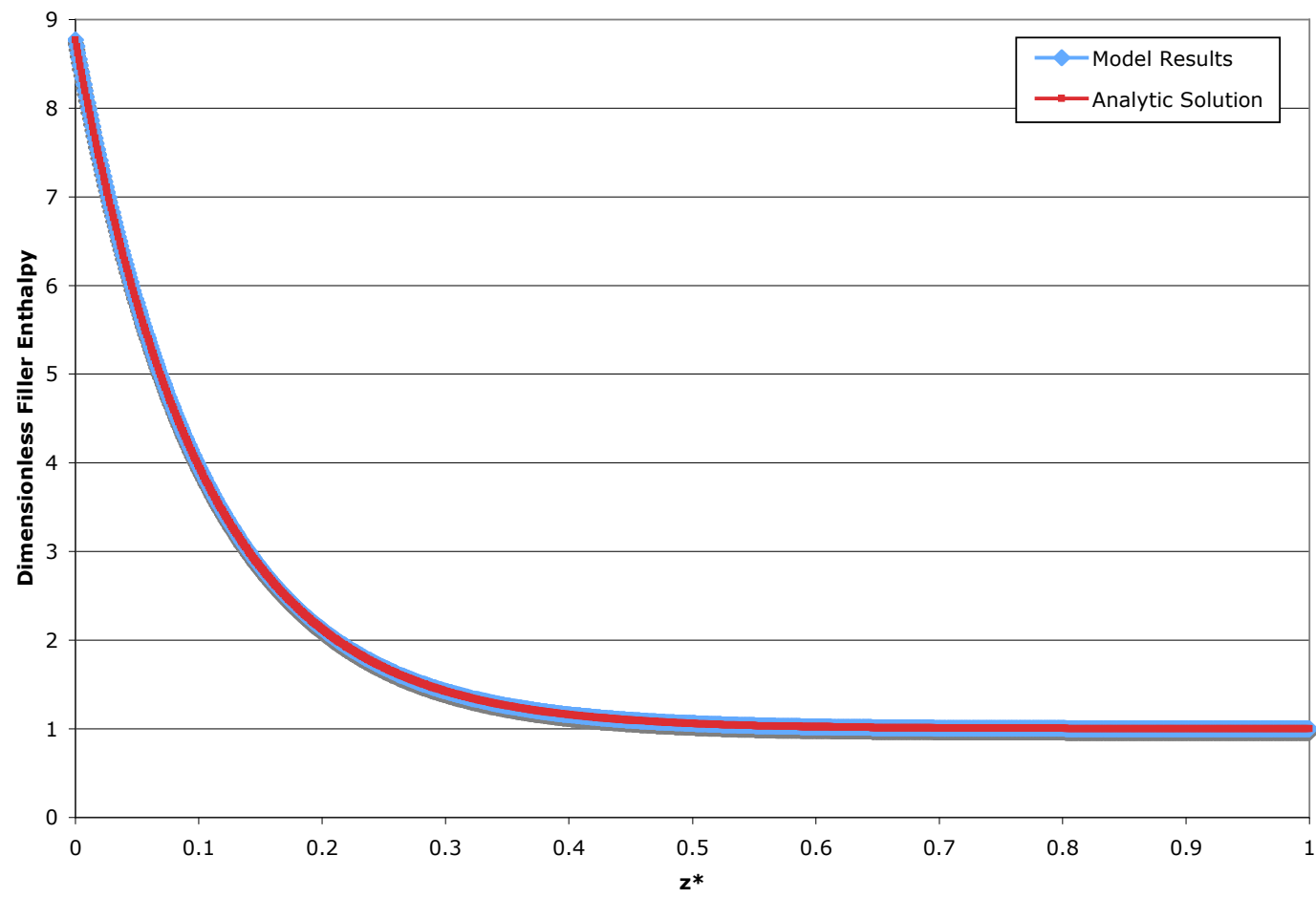

Fig. 8 Model and Analytic Solution Comparison of Filler Enthalpy in Filler Melting Region 
Another step for verification of the model features comparison to the previously constructed model by Van Lew [10]. Though the previous work did not take a PCM filler into account, comparison of filler and fluid temperatures could still be made for a filler in solid phase state. Running an identical case (from the original boundary verification above) for the two models and comparing results at each numerical grid at the last instance in time, using the Van Lew results as a reference, the maximum percentage errors were found to be $0.000861 \%$ and $0.000738 \%$ per PCM filler and fluid temperature, respectively. These very small deviations were likely due to the nature of input to the models, mixed with standard numerical round-off error. The newly developed model took properties as input, and calculates the corresponding dimensionless parameters from them, while Van Lew's model simply took the dimensionless parameter values as input directly. This was clearly a source of small error. However, we saw the minute deviation is already of the same magnitude as the numerical round off seen in previous verifications. Thus, the additional significant figures in accurate dimensionless parameter calculation were not necessarily useful.

Finally, comparison to experimental results by Nallusamy et al. [3] was also made to demonstrate the validity of our numerical scheme. They used encapsulated spherical capsules of paraffin with melting temperature at $60{ }^{\circ} \mathrm{C}$ as the PCM and water as the heat transfer fluid. The inlet fluid temperature was maintained at $70{ }^{\circ} \mathrm{C}$ and the mass flow rate was fixed at 2 liters per minute. Using their experimental conditions and properties given, we estimated the governing parameters.

$$
\begin{aligned}
& H_{C R}=1.008 \\
& \tau_{r}=1.0269 \\
& \theta_{f_{\text {inlet }}}=1 \\
& \theta_{r_{O}}=\theta_{f_{O}}=0 \\
& \theta_{r_{-} \text {melt }}=0.7368 \\
& \Delta t^{*}=\Delta z^{+}=0.001
\end{aligned}
$$

Figures 9 and 10 shows the comparisons of the fluid temperature and the PCM temperature as a function of time located at the middle of the tank, respectively. It can be observed that the results are in good agreement. 


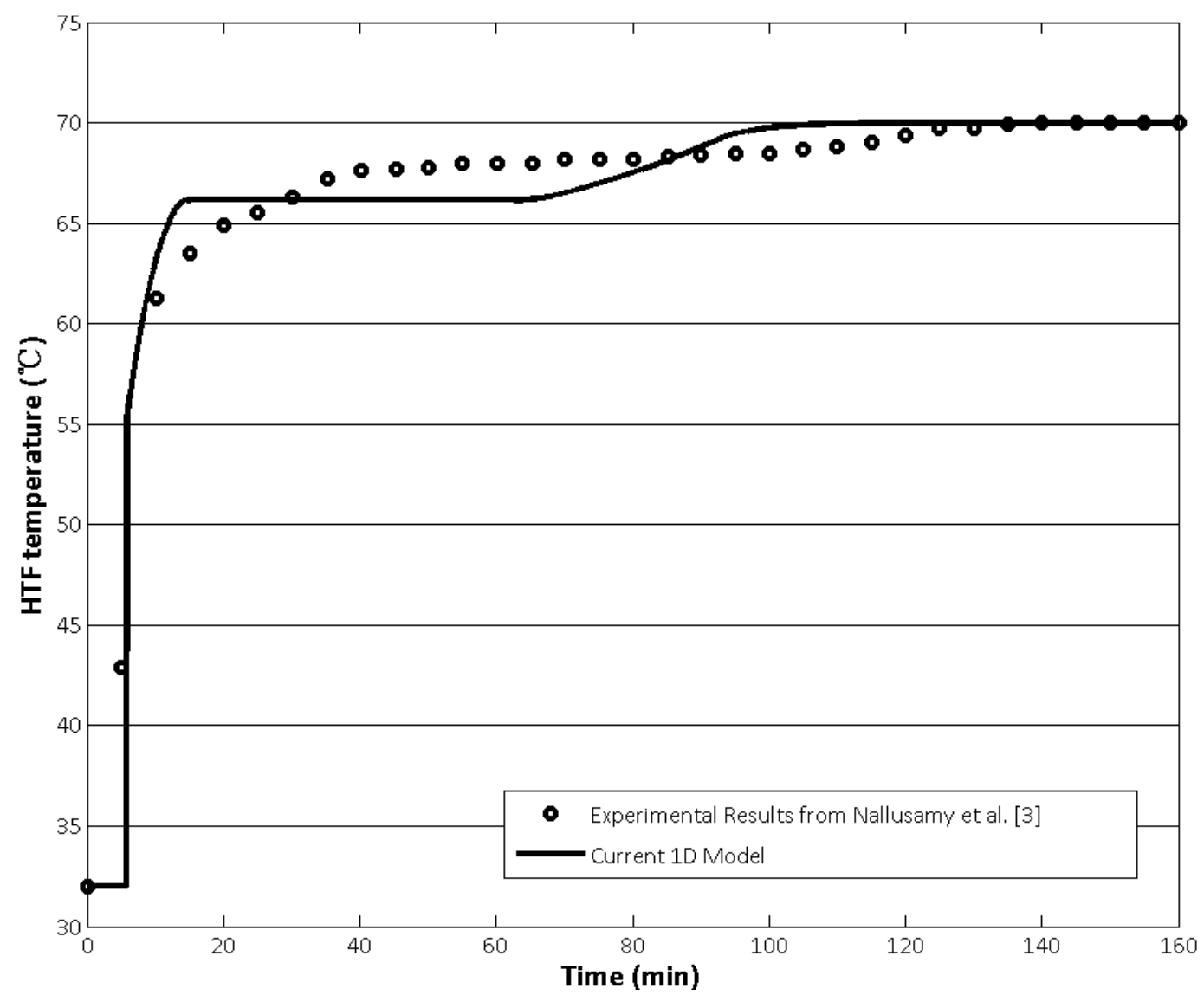

Fig. 9 Comparison of Fluid Temperature Located at the Middle of the Tank 


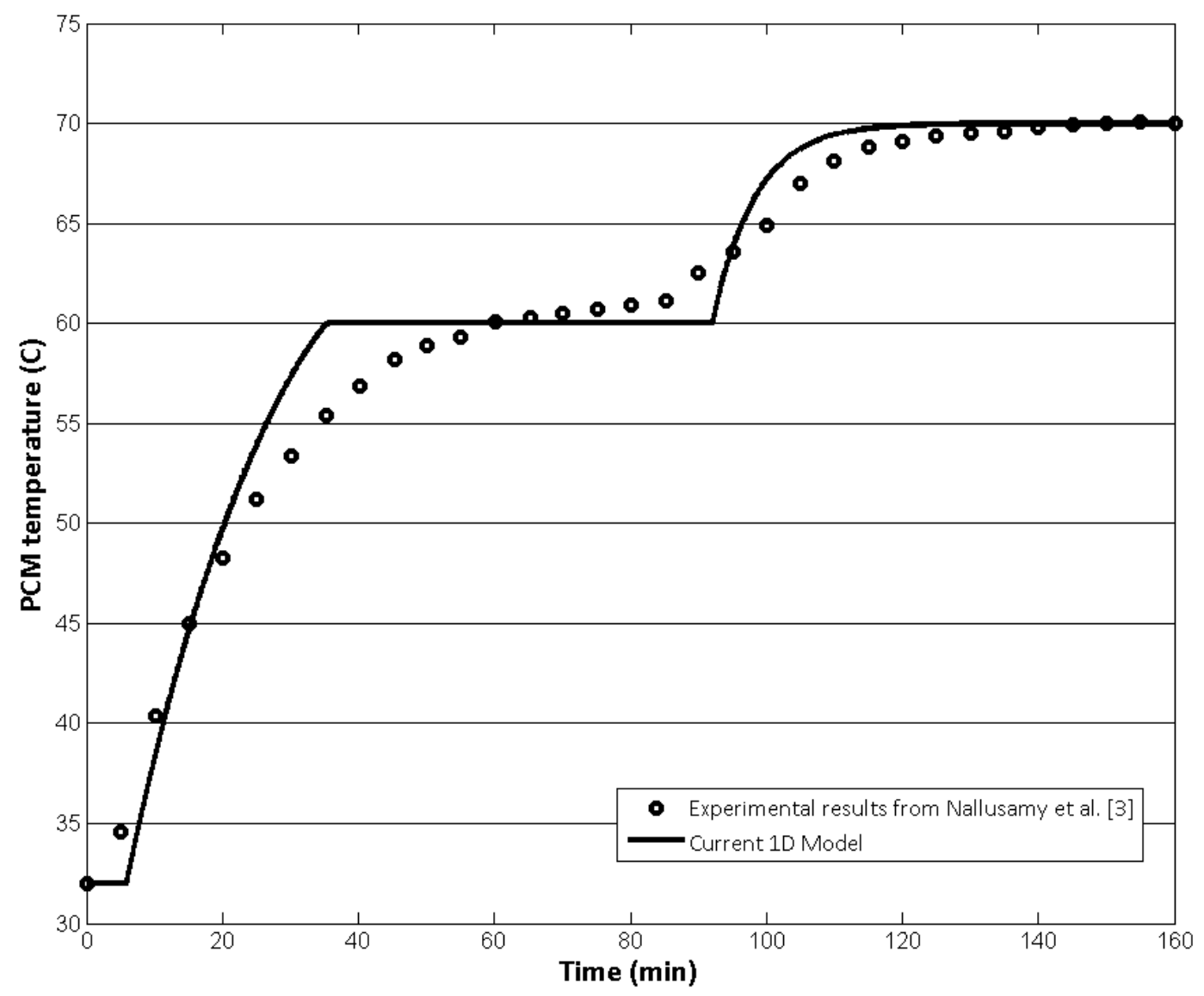

Fig. 10 Comparison of PCM Temperature Located at the Middle of the Tank

\section{Model Application}

With the new model developed and verified, we briefly show some of its capabilities in application. Having expanded considerations to a PCM filler, we first outline a simple charge/discharge process for a thermocline utilizing this sort of material. We begin with the charge process, with key numerical parameters as below.

$$
\begin{aligned}
& H_{C R}=0.5785 \\
& \tau_{r}=0.1117 \\
& \theta_{f_{\text {illet }}}=1 \\
& \theta_{r_{O}}=\theta_{f_{O}}=0 \\
& \theta_{r_{-} \text {melt }}=0.5 \\
& \Delta t^{*}=\Delta z^{+}=0.001
\end{aligned}
$$

Heating occurred from the initial state to a final dimensionless time of 4.0, for which temperature profiles are again displayed at dimensionless times 0, 0.1, 0.5, 1.0, 2.0, 3.0, and 4.0. These can be viewed in Fig. 11 and Fig. 12, per the fluid and PCM filler, respectively. 


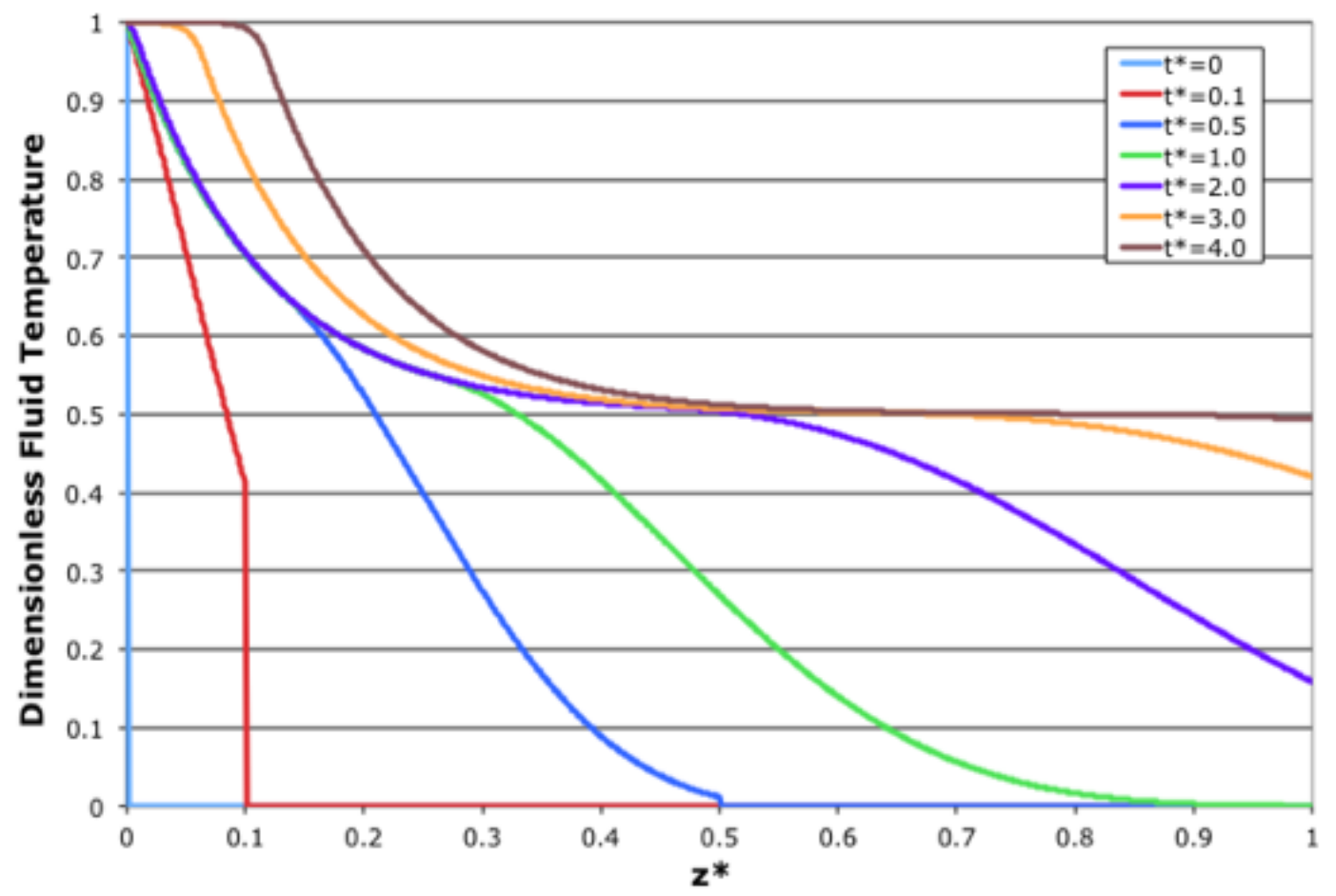

Fig. 11 Fluid Temperature Profiles at various instance of time for Charge Process of PCM Filler

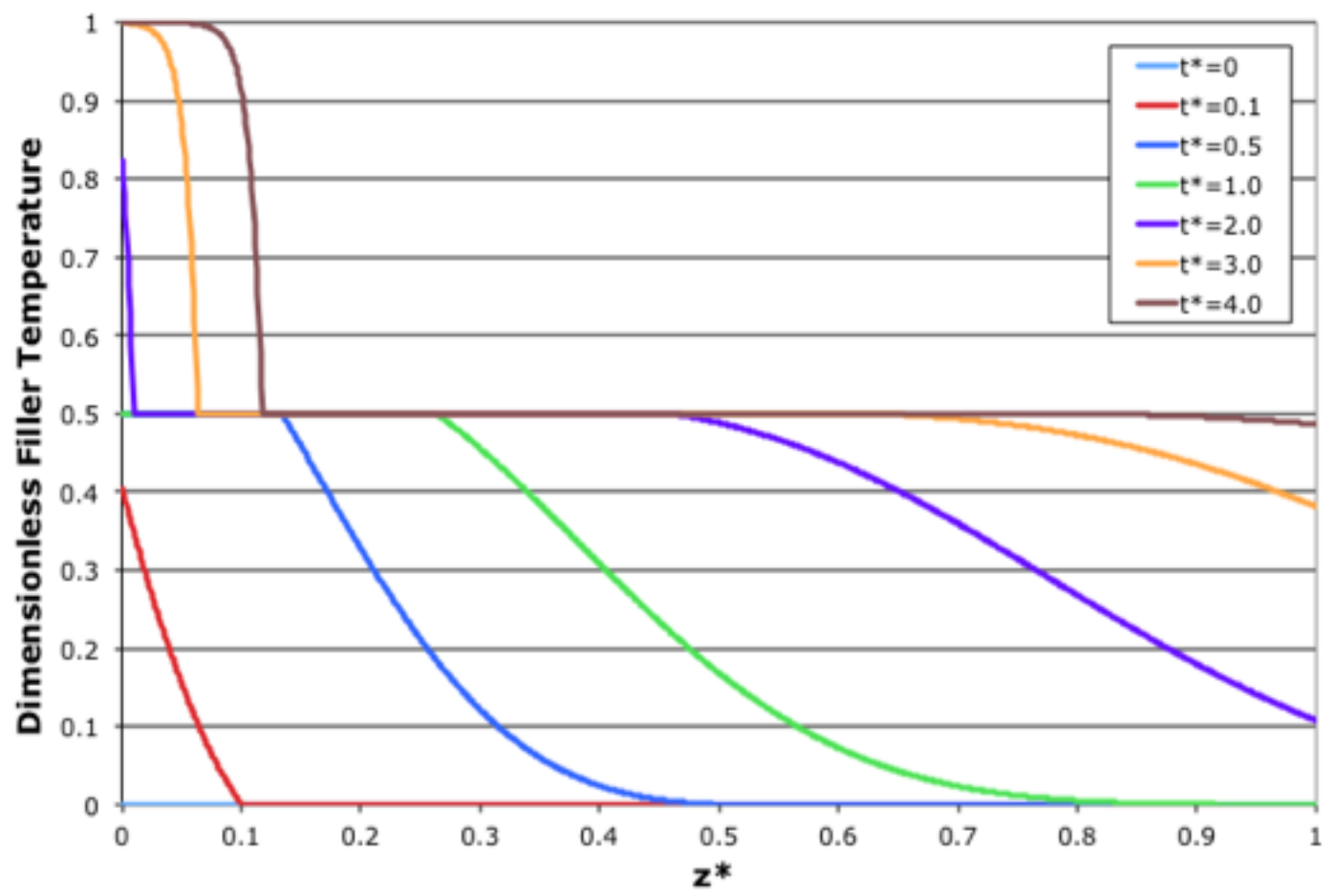

Fig. 12 Filler Temperature Profiles at various instance of time for Charge Process of PCM Filler 
Analysis of the results offers much insight on the material behavior in the tank during the process. With the time allotted, heat at the boundary raised the PCM filler temperature, melted it fully, and continued heating it in its liquid phase state. We see the PCM filler first started transitioning into its melting phase state soon after dimensionless time 0.1 , and then finally collected enough energy to continue to a liquid PCM filler just before dimensionless time 2.0.

In space, recalling the PCM filler melting temperature of 0.5 , the positioning of the corresponding PCM filler phase states became quite clear. At the end of the charge, with PCM filler temperature at the outlet remaining just below the PCM filler melting temperature, heat propagation sent the solidus phase state interface near the end of the tank, leaving only a small amount of solid PCM filler near the outlet. The constant filler temperature of 0.5 throughout most of the space shows the large latent heat maintaining the PCM filler in this intermediate melting phase state. Finally, near the inlet, the temperature of the PCM filler finally rose above 0.5 , signifying a liquid PCM filler, and placing the liquidus phase state interface near this inlet boundary. We review the interface positions as functions of time in Fig. 13 below to confirm this behavior.

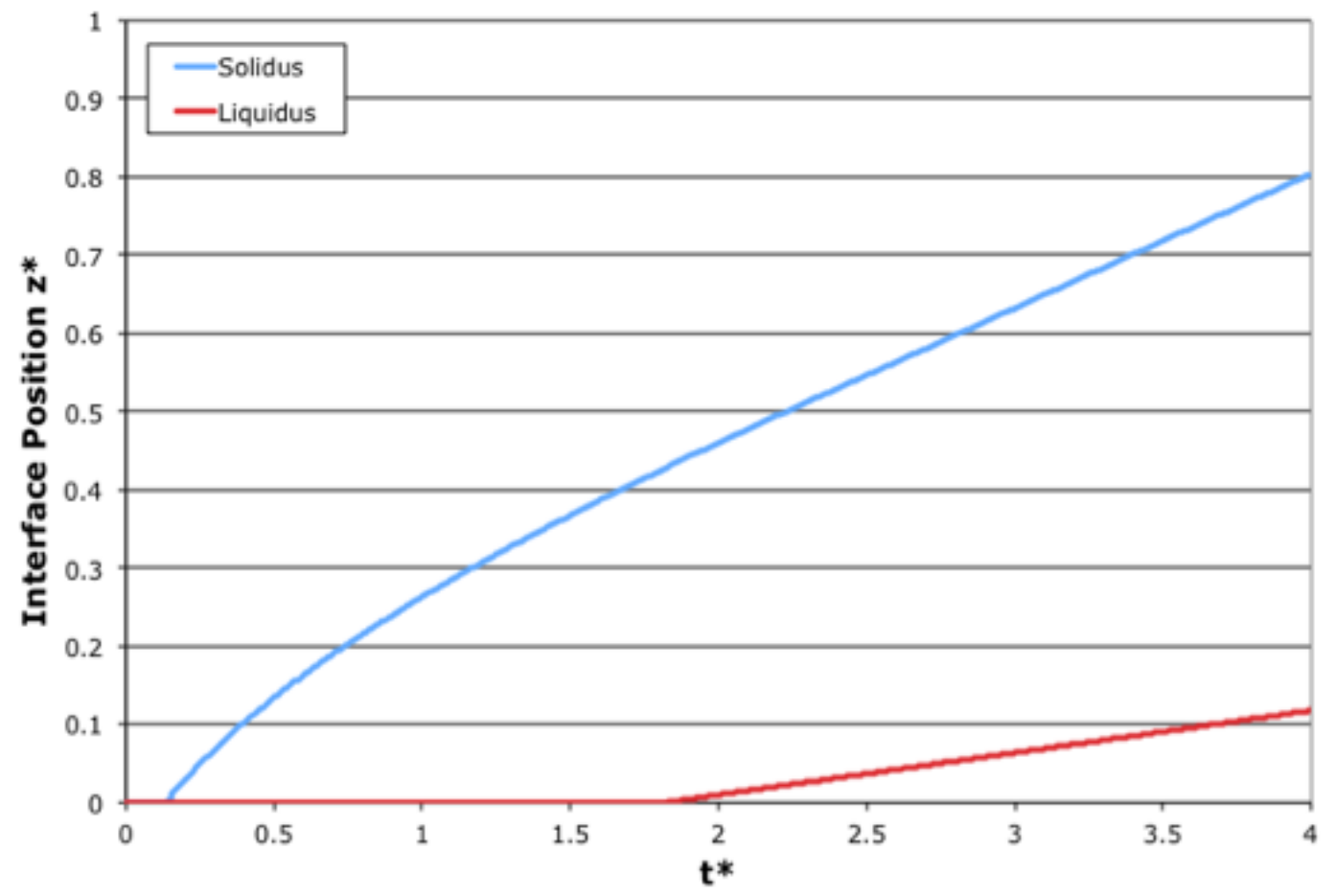

Fig. 13 Phase Change Interface Positions as Functions of Time for Standard Charge Process of PCM Filler

The plot confirms what is seen in the temperature distributions. Melting occurred relatively soon after heating began, corresponding to the start of the solidus phase state interface. With continued heating, this interface traveled through space, and is left near the end of the tank. Late in the charging cycle, enough heat had been put in to produce a fully liquid PCM filler, corresponding to the beginning of a liquidus phase state interface. With only little time left in the process, this second interface traveled only slightly into the space. Discontinuities in the fluid 
temperature profiles are again seen before dimensionless time 1.0 for the same reasons stated in the non-melt PCM filler case. In Fig. 14, the corresponding liquid fraction vs $\mathrm{z}^{*}$ at $\mathrm{t}^{*}=0,0.5,1.0,2.0,3.0$, and 4.0 is presented. It varies between 0 (solid) to 1 (liquid). At $t^{*}=0.5$, the melt region is from $\mathrm{z}^{*}=0$ to 0.135 and we see that this region is partially melt. As time advance, the melt region expands and the liquid fraction increases. At $t^{*}=2.0$, we see that the PCM near the entrance has been fully melted. The liquid region continues to grow as time advance.

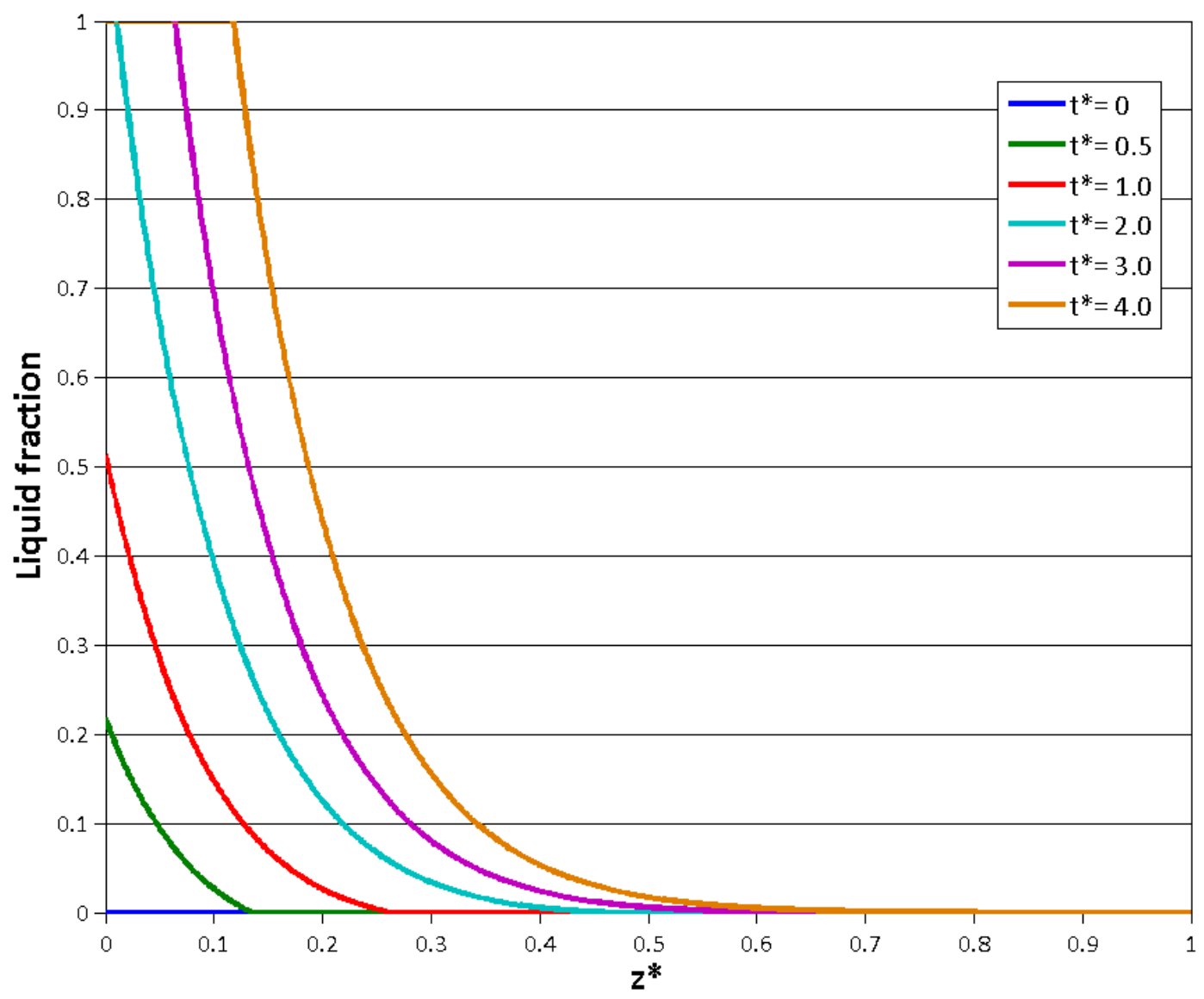

Fig. 14 Liquid Fraction Profiles at various instance of time for Standard Charge Process of PCM Filler

Post charge process, we review how the tank achieves equilibrium to extract the effects of having a PCM filler. An equilibration calculation was applied to the final fluid and PCM filler temperature profiles before turning fluid flow around and discharging the stored energy. The final profiles of the tank, along with their corresponding equilibrium profile, can be viewed below in Fig. 15. 


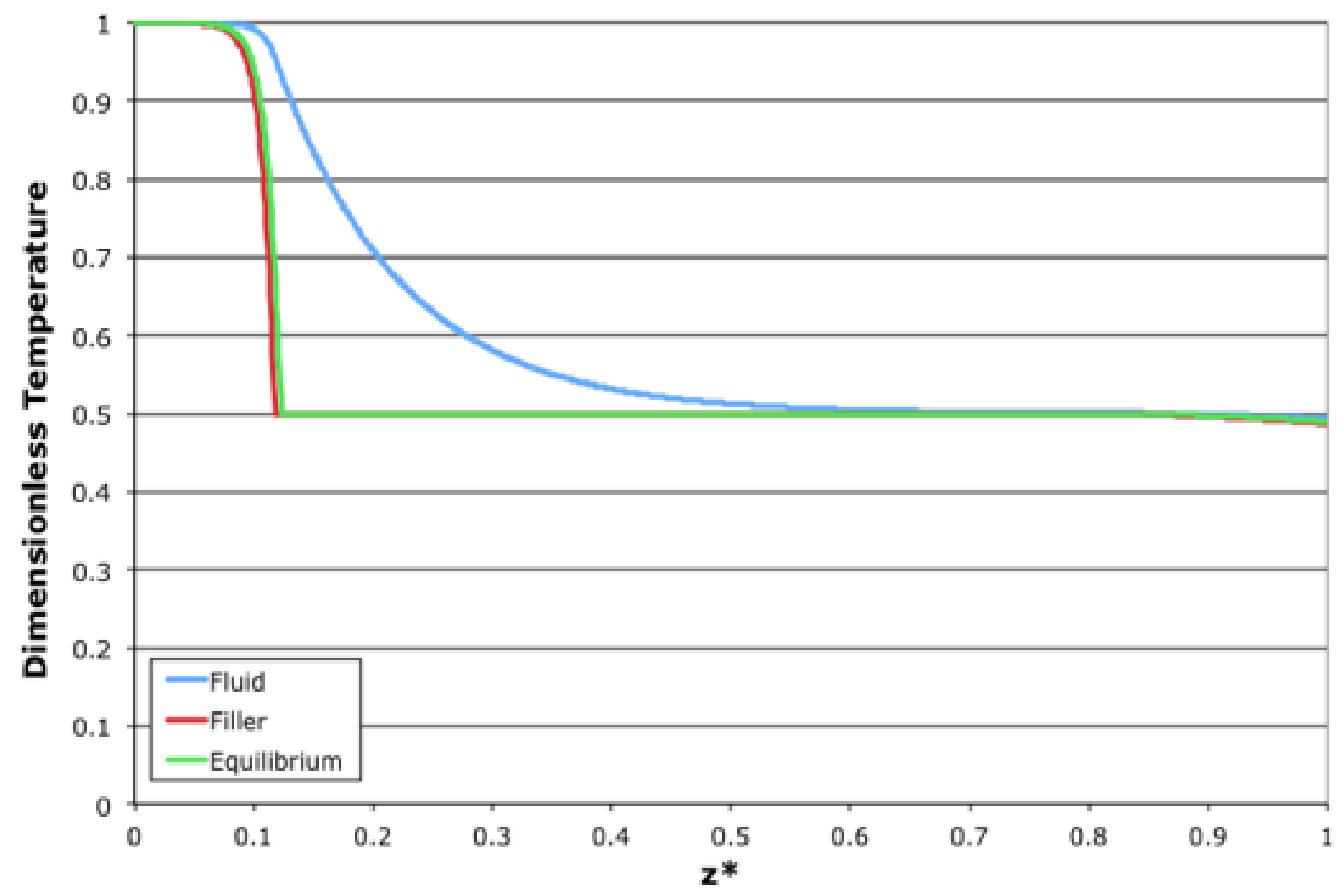

Fig. 15 Equilibration of Final Temperature Profiles after Charge Process for PCM Filler

Reviewing the results, we see the effects of latent heat of equilibration results. With the end of charge profile featuring mostly melting PCM filler, the temperature difference between fluid and PCM filler was not enough to lift the PCM filler above its melting temperature. It was only close to the phase state interfaces, and in the solid and liquid PCM filler phase state regions, that the two profiles found a more intermediate position, though they were originally so close that the change is very small. Thus, we saw a larger reduction in a relatively wide portion of the fluid temperature profile from equilibration, leading to a larger decrease of fluid output temperature for eventual energy production.

With equilibrium applied, the resulting temperature profile became the initial fluid and PCM filler condition for the discharge process. The tank was cooled at the inlet by the same constant low fluid temperature as the nonmelting PCM filler case, and key numerical parameters remain the same. Discharge was again run to a final dimensionless time of 4.0, for which the tank profiles are displayed at dimensionless time values 0, 0.1, 0.5, 1.0, 2.0, 3.0, and 4.0. Results can be viewed in Fig. 16 and Fig. 17, per the fluid and PCM filler, respectively. 


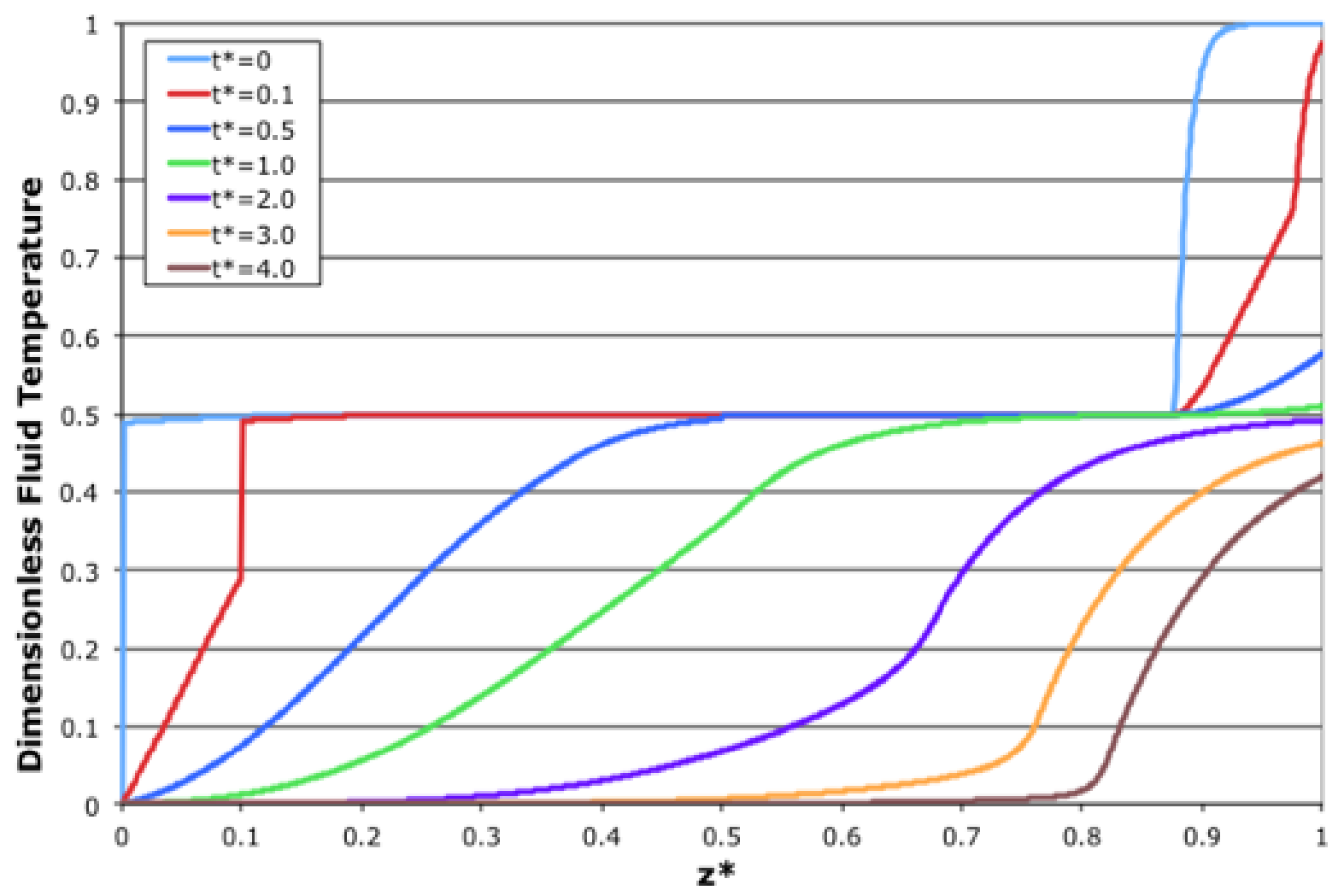

Fig. 16 Fluid Temperature Profiles at various instance of time for Discharge Process for PCM Filler

For this process, the profiles behaved in a reverse order as the charge. Cooling brought the PCM filler temperature down, reducing the amount of liquid PCM filler, and increasing the amount of solid PCM filler. Just after dimensionless time 1.0, cooling sent the liquidus phase state interface out of the storage tank, thus removing all liquid PCM filler. Heat extraction continued until the solidus phase state interface traveled past the position 0.8 , leaving mostly solid PCM filler, with a comparatively small amount of melting PCM filler. The fluid temperature profiles followed as expected, with discontinuities between inlet affected fluid and initial state seen before dimensionless time 1.0, as before. 


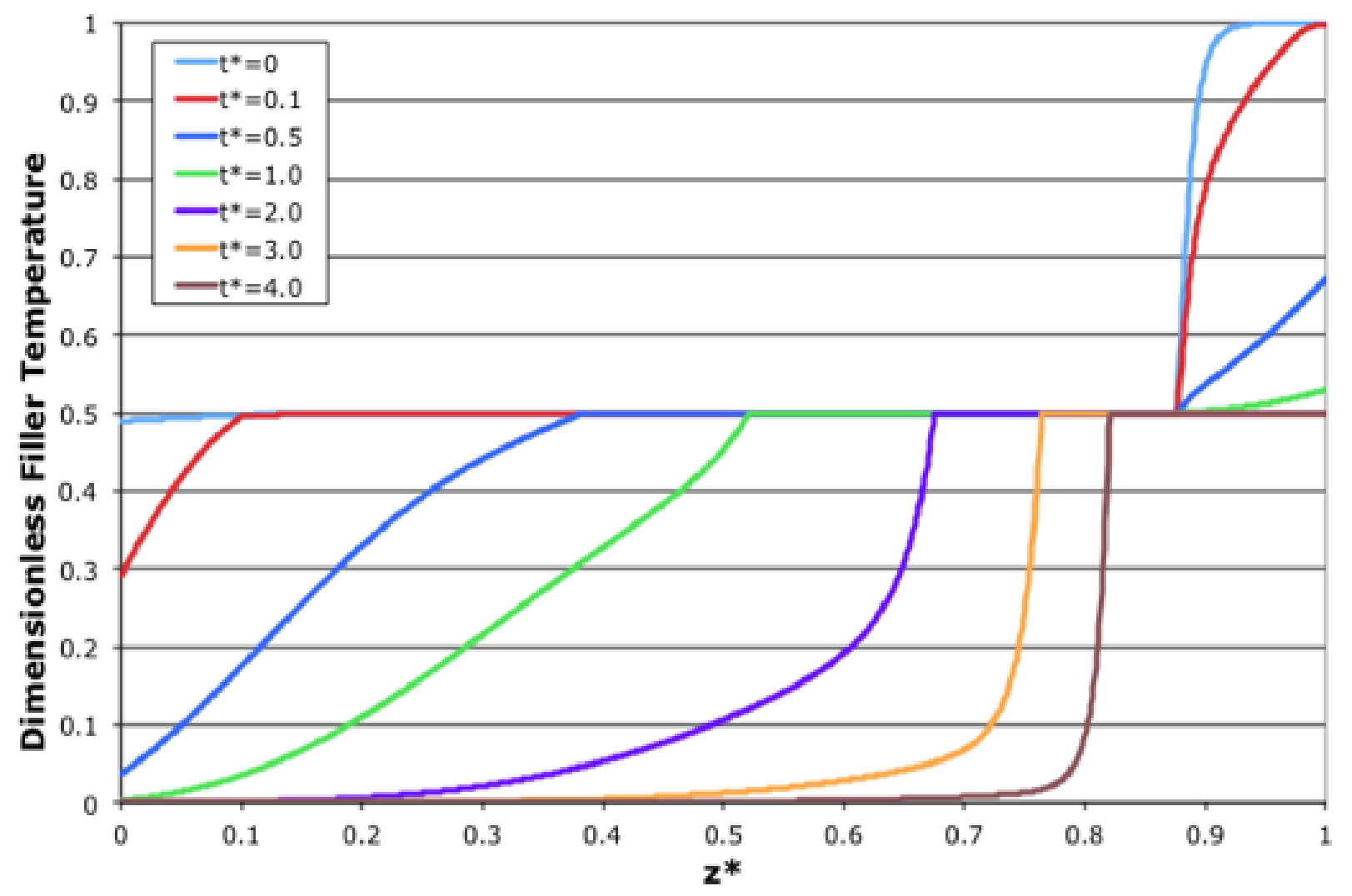

Fig. 17 Filler Temperature Profiles at various instance of time for Discharge Process for PCM Filler

Finally, we showcase the versatility of the model by applying a variable boundary condition. With it, we aimed to apply heat to the PCM filler, forcing phase change(s), at which time the boundary condition was reversed, removing heat to produce an additional phase state interface reverting to the earlier phase state(s). Seeing satisfactory modeling in a complex heat transfer case such as this will confirm the general consideration implemented. The model was run as a charge process until a final dimensionless time equal to 5.0. Key properties were as follows.

$$
\begin{aligned}
& H_{C R}=0.5725 \\
& \tau_{r}=0.1174 \\
& \theta_{r_{o}}=\theta_{f_{O}}=0 \\
& \theta_{r_{-} \text {melt }}=0.1
\end{aligned}
$$

with inlet fluid temperatures as below. 


$$
\theta_{f_{-} \text {inlet }}=\left\{\begin{array}{lll}
1.0 & 0 \leq t^{*}<1.5 \\
0.6 & & 1.5 \leq t^{*}<2.5 \\
& \text { for } & 2.5 \leq t^{*}<4.0 \\
0.3 & & 4.0 \leq t^{*}<5.0
\end{array}\right.
$$

This boundary condition represented a discontinuous series of constant fluid temperature values applied over specific amounts of time. Heating was applied quickly to produce two-phase state interfaces, and then a gradually stepped cooling was used to revert to a previous phase state. Below, we trace the temperature profile evolutions throughout the various changes in boundary condition.

We first look at the temperatures within the tank at dimensionless time 1.499, which is the last instance of inlet fluid condition equal to 1.0. This can be viewed in Fig. 18. The profiles produced represent a simple constant inlet PCM filler temperature charge. Heating produced two-phase state interfaces, leaving 3 separate phase states of PCM filler in the tank. Though paraffin features a large latent heat, the large temperature gradient left above the melting temperature due to the high inlet fluid temperature heated through the melting PCM phase state relatively quickly. We now continue to the distributions at dimensionless time 2.499, Fig. 19, which is the last instance in time with the inlet temperature of 0.6 as the boundary condition.

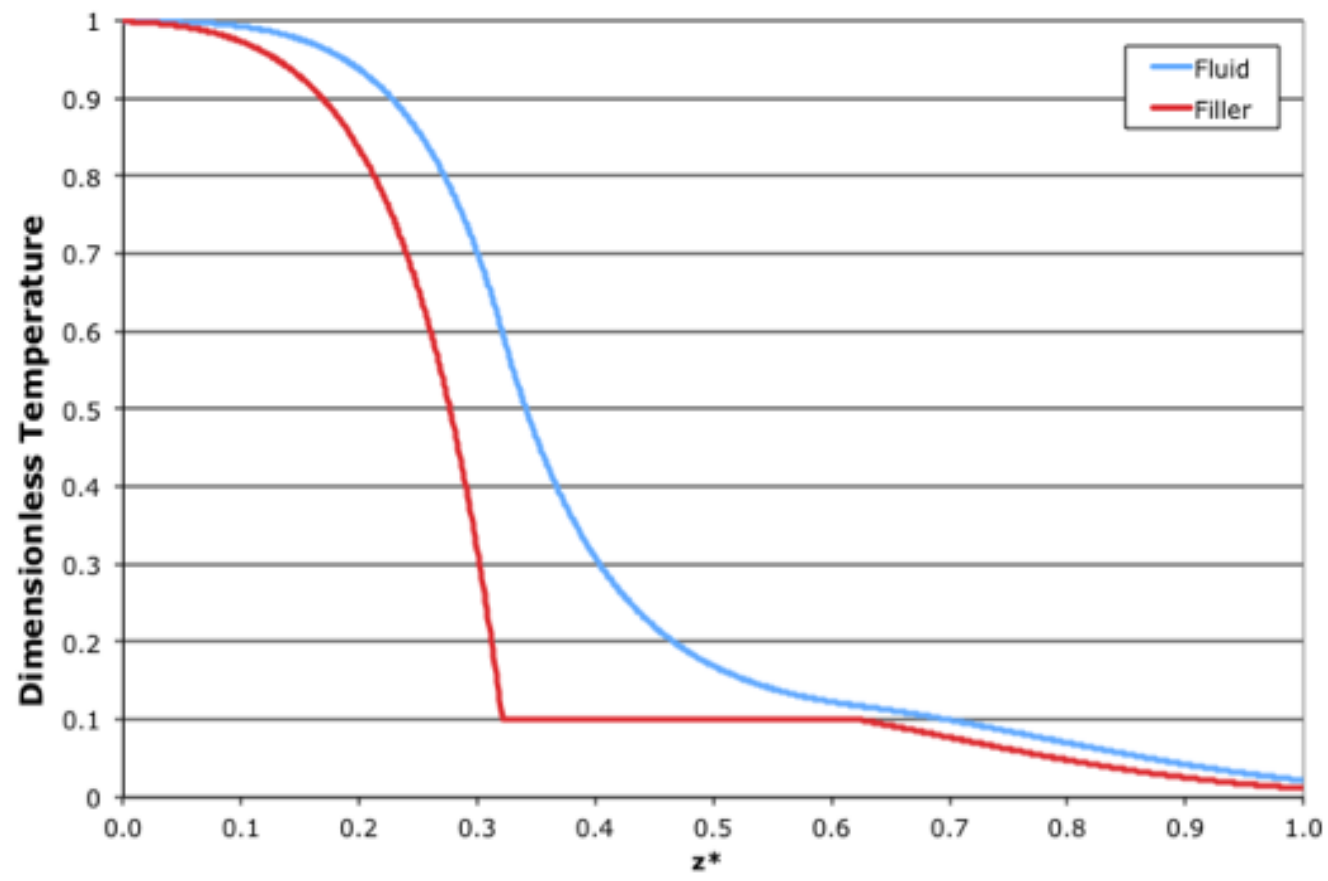

Fig. 18 Fluid and Filler Temperature Profiles at dimensionless time 1.499 


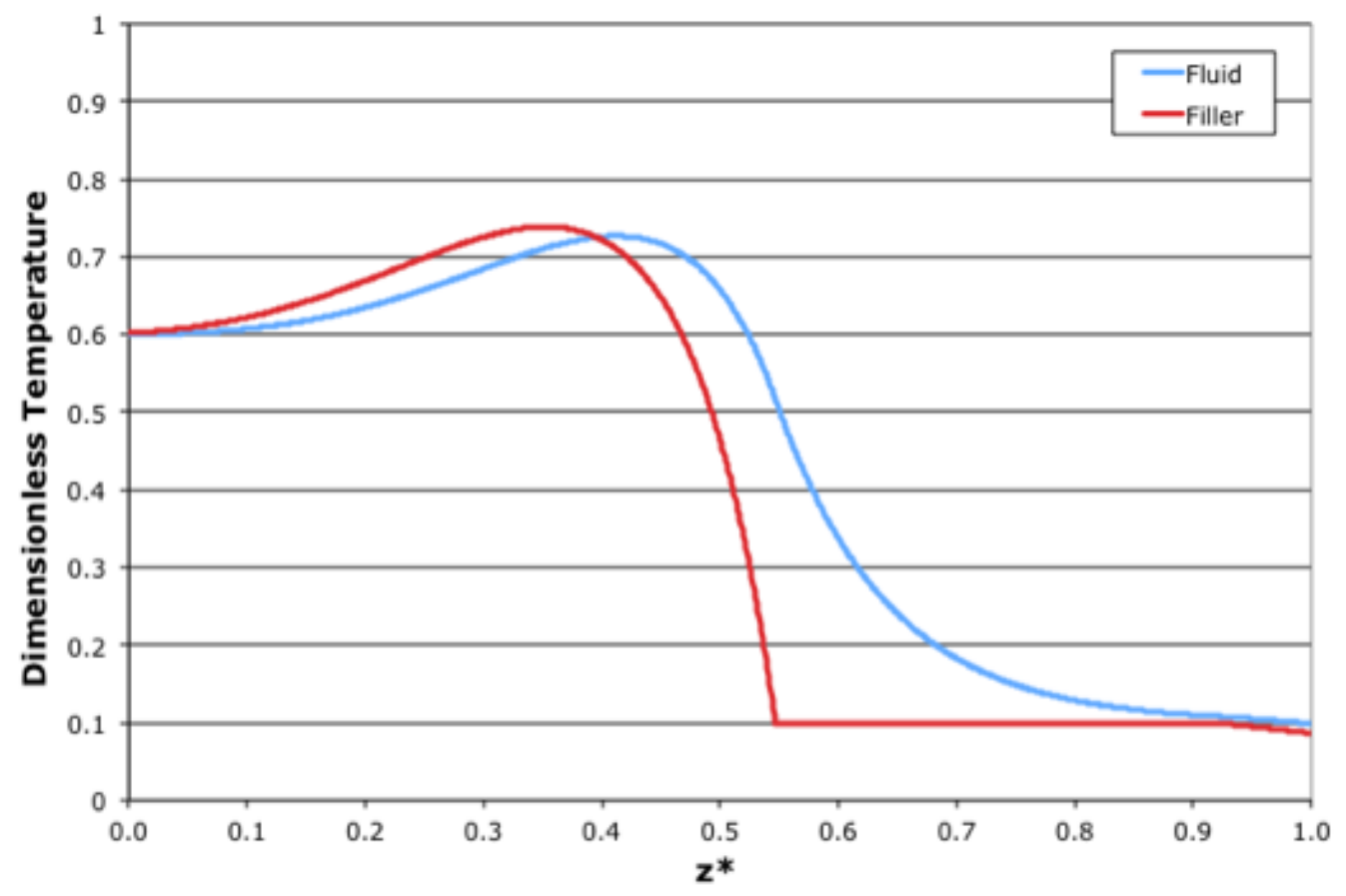

Fig. 19 Fluid and Filler Temperature Profiles at dimensionless time 2.499

At this instance in time, we see an interesting response in the profiles. Due to the wide spread of PCM filler temperature after the previous heating, the new inlet condition worked to both cool and heat, depending on the inlet fluid's relation to the temperatures within the tank at a certain position. The effect was seen throughout the entire tank, as this new condition, traveling at the characteristic speed, was able to traverse the entire space in the $\Delta t^{*}=1.0$ it was applied. When interacting with the high temperatures towards the tank inlet, we saw a cooling that brings both PCM filler and fluid profiles gradually down to the inlet temperature condition. Further in space, where tank temperature values remain lower, the new condition continued to heat the tank profiles. The two heat transfer types met in a continuous parabolic fashion, representing the eventual change of liquid PCM filler being heated to liquid PCM filler experiencing cooling. Heat propagation through the tank had also facilitated the propagation of the two previously mentioned interfaces, as expected. The initial solidus phase state interface was found very close to the outlet, leaving little solid PCM filler in the tank, while the creation of more liquid PCM filler placed the liquidus phase state interface past the midway point in the tank.

Past this time, another lower fluid boundary temperature was applied up until the dimensionless time 3.999. The profiles at this time can be viewed in Fig. 20. As expected, this new inlet fluid temperature dropped temperature profiles even lower. All solid PCM filler has now melted, meaning the solidus phase state interface has left the space at some time during the application of this inlet condition. Conditions had also produced even more liquid PCM filler, and correspondingly shifted the liquidus phase state interface further towards the outlet. Finally, the last drop in boundary temperature was applied from dimensionless time 4.0 through the end of the run. The resulting end temperature profile can be viewed in Fig. 21. 


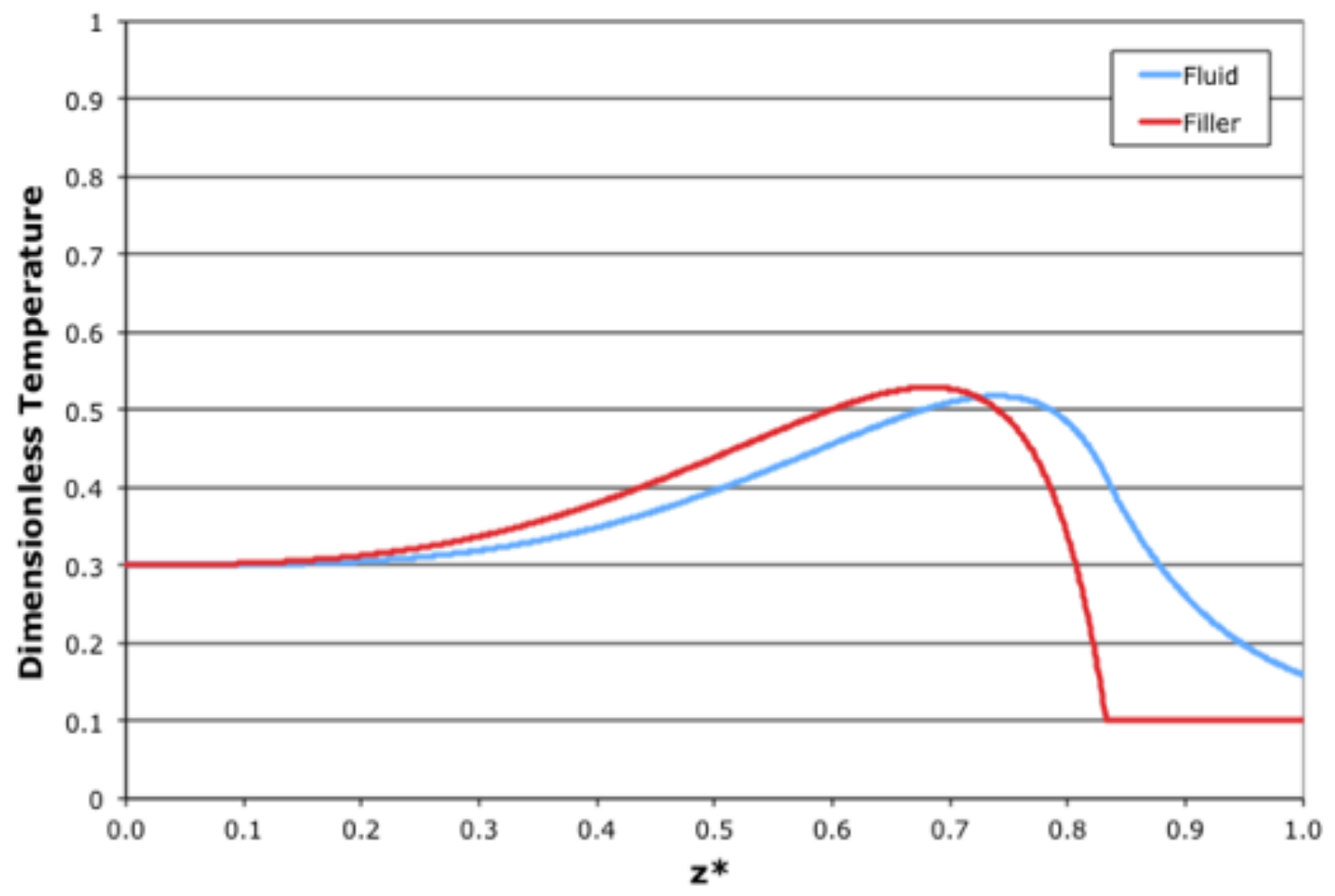

Fig. 20 Fluid and Filler Temperature Profiles at dimensionless time 3.999

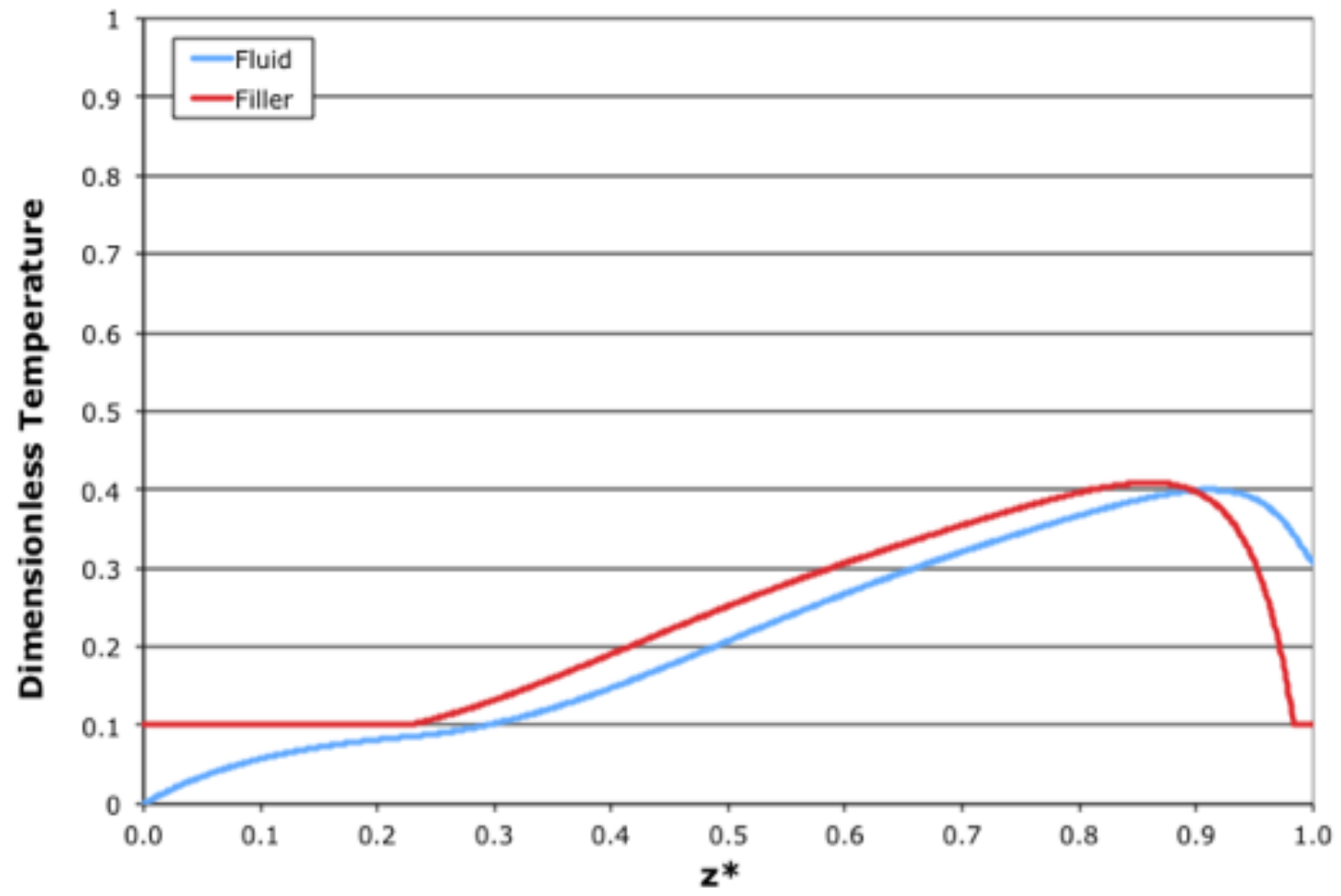

Fig. 21 Fluid and Filler Temperature Profiles at dimensionless time 5.0 
With this inlet fluid temperature, the system saw the lowest cooling temperature in the run. The PCM filler temperature was even further decreased, eventually returning the PCM filler at the boundary back to a melting PCM filler, and creating a second liquidus phase state interface. The original melting PCM filler was almost all fully liquefied, meaning we expected the first liquidus phase state interface to be very close to the tank outlet. This left three unique phase states in the tank from input to output- melting, liquid, and melting. To complete understanding of the process, we compiled the travel of interfaces throughout. This can be viewed below, Fig. 22.

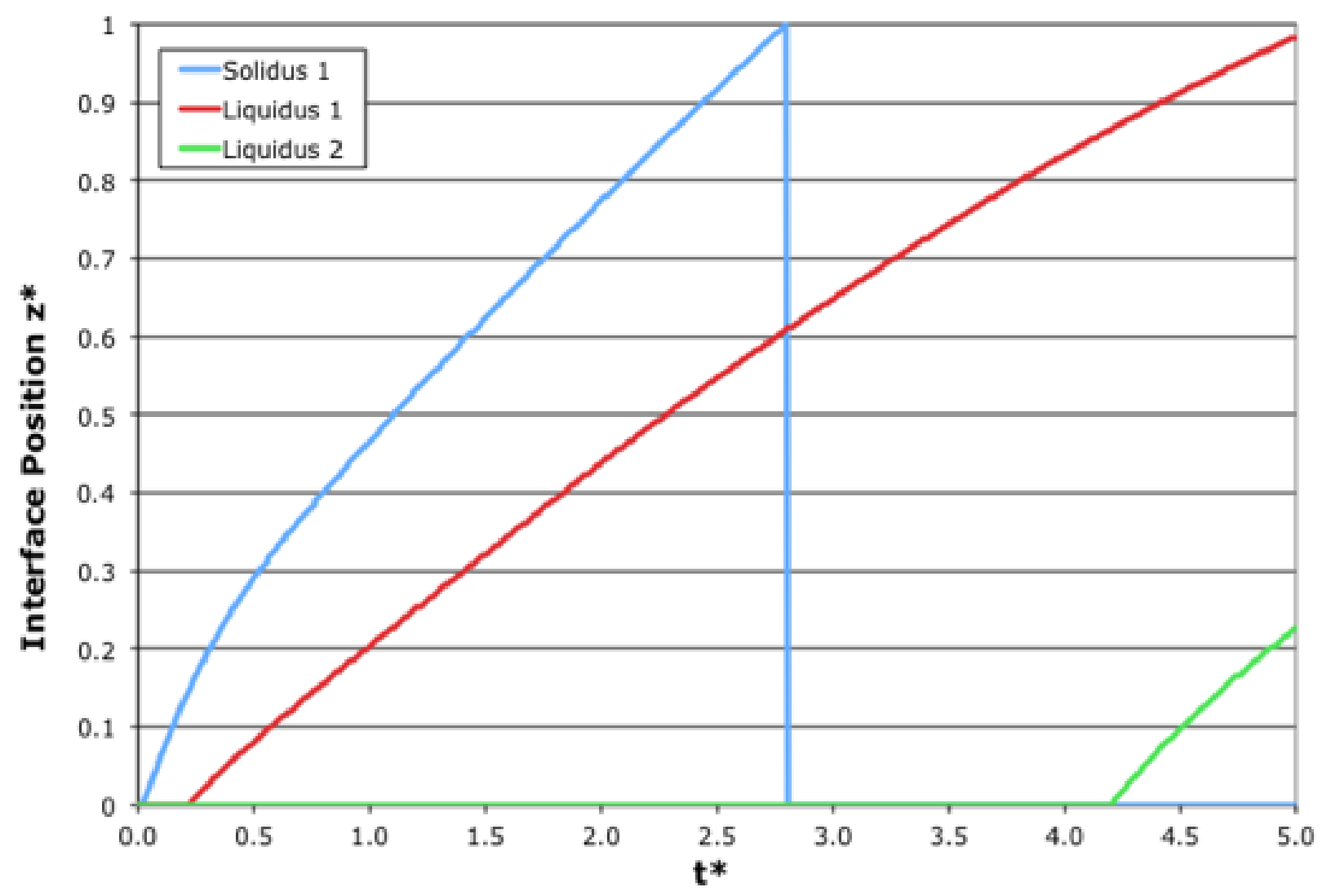

Fig. 22 Phase Change Interface Positions as Functions of Time for Variable Boundary Charge Process

The interface travel confirms what was outlined in the profiles above. The first phase state interface, of solidus type, was created early in the heating. Slightly past the halfway mark of the process, this phase state interface traveled far enough to leave the space entirely, taking all solid PCM filler with it. After heating through the latent region of melting PCM filler, the first liquidus phase state interface was created, bringing with it liquid PCM filler to the tank. This phase state interface traveled nearly the entire space, and turned nearly all the remaining melting PCM filler into liquid PCM filler. Finally, after adequate cooling, a second liquidus phase state interface was created as the liquid PCM filler began returning to a melting PCM filler. This interface saw little travel, as this phase change occurred near the end of the run. Thus, throughout the entire case, heating and cooling created 3 unique phase state interfaces, all of which were tracked by the model. Interface travel was clearly non-linear, though speed decreased to 
a near constant rate as the temperature difference between fluid and PCM filler driving their travel decreased with time.

\section{Conclusion}

Use of the method of characteristics has once again produced an accurate and efficient model. Temperature profiles and interface travel from both charge and discharge processes produced by the model showed no oddities, and behaved as expected. Additionally, extreme diligence with considerations of interface interactions with the numerical characteristics in all possible heating conditions allowed for fully versatility while retaining accuracy through variable phase states. This was verified by choice analytical solutions; and, most importantly, a trial of variable boundary condition, where three phase state interfaces were created through both heating and cooling inlet conditions in a single computation. In all, the model could handle variable inlet conditions, any amount of phase changes, and an initial condition with any amount of filler phase states. This way, there are virtually no restriction in the properties and criterion for thermocline operation that one could model with it in the future. Because of the wave nature of the governing equation, method of characteristics is very well suited. It provides fast, robust, and highly accurate solutions. Typical charging and discharging cases take a few seconds on a $2.5 \mathrm{GHz}$ Intel Core 2 Duo processor. Parametric studies are being done and will be report in the near future.

\section{Acknowledgment}

The authors are grateful for the partial supports from the U.S. Department of Energy and National Renewable Energy Laboratory under DOE Award: DE-FC36-08GO18155 and the Idaho National Laboratory under award number 00095573.

\section{References}

[1] N. Ukrainczyk, S. Kurajica, Thermophysical Comparison of Five Commercial Paraffin Waxes as Latent Heat Storage Materials. Chemical and Biochemical Engineering Quarterly 24.2 (2010) 129-137.

[2] R. J. Krane, M. J. M. Krane, The optimum design of stratified thermal energy storage systems-Part II: Completion of the analytical model, presentation and interpretation of the results. Journal of Energy Resources Technology 114.3 (1992) 204-208.

[3] N. Nallusamy, S. Sampath, R. Velraj, Experimental investigation on a combined sensible and latent heat storage system integrated with constant/varying (solar) heat sources. Renewable Energy 32 (2007) 1206-1227.

[4] P. Verma, Varun, S. K. Singal, Review of Mathematical Modeling on Latent Heat Thermal Energy Storage Systems using Phase-Change Material. Renewable and Sustainable Energy Reviews, 12 (2008), 999-1031.

[5] F. L. Tan, S. F. Hosseinizadeh, J. M. Khodadadi, Liwu Fan, Experimental and computational study of constrained melting of phase change materials (PCM) inside a spherical capsule. International Journal of Heat and Mass Transfer 52.15 (2009) 3464-3472. 
[6] Weihuan Zhao, A. F. Elmozughi, A. Oztekin, S. Neti, Heat transfer analysis of encapsulated phase change material for thermal energy storage. International Journal of Heat and Mass Transfer 63 (2013) 323-335.

[7] T. E. Schumann, W. Heat transfer: A Liquid Flowing through a Porous Prism. Journal of the Franklin Institute 208.3 (1929) 405-416.

[8] J. E. Pacheco, S. K. Showalter, W. J. Kolb, Development of a molten-salt thermocline thermal storage system for parabolic trough plants. ASME Journal of Solar Energy Engineering 124.2 (2002) 153-159.

[9] A. Shitzer, M. Levy, Transient Behavior of a Rock-Bed Thermal Storage System Subjected to Variable Inlet Air Temperatures: Analysis and Experimentation. ASME Journal of Solar Energy Engineering 105.2 (1983) 200-206.

[10] J. Van Lew, P.W. Li, C.L. Chan, W. Karaki, J. Stephens, Analysis of heat storage and delivery of a thermocline tank having solid filler material. ASME Journal of Solar Energy Engineering 133.2(2011) 021003.

[11] A. Felix Regin, S.C. Solanki, An Analysis of a Packed Bed Latent Heat Thermal Energy Storage System using PCM capsules: Numerical Investigation. Renewable Energy 34 (2009) 1765-1773.

[12] S. Wu, G. Fang, X. Liu, Dynamic Discharging Characteristics Simulation on Solar Heat Storage System with Spherical Capsules using Paraffin as Heat Storage Material. Renewable Energy 36 (2011) 1190-1195.

[13] K. Nithyanandam, R. Pitchumani, Thermal energy storage with heat transfer augmentation using thermosyphons. International Journal of Heat and Mass Transfer 67 (2013) 281-294.

[14] K. Nithyanandam, R. Pitchumani, Computational studies on a latent thermal energy storage system with integral heat pipes for concentrating solar power. Applied Energy, 103 (2013) 400-415.

[15] A. R. Archibold, M. M. Rahman, D. Y. Goswami, E. L. Stefanakos, Parametric Investigation of the Melting and Solidification Process in an Encapsulated Spherical Container. Proceedings of the ASME 2012 6th International Conference on Energy Sustainability. July 23-26, 2012, San Diego, CA, USA

[16] N. R. Vyshak, G. Jilani, Numerical analysis of latent heat thermal energy storage system. Energy Conversion and Management 48.7 (2007) 2161-2168.

[17] J. Van Lew, P.W. Li, C.L. Chan, W. Karaki, J. Stephens, Transient heat delivery and storage process in a thermocline heat storage system. Proceedings of ASME 2009 International Mechanical Congress and Exposition, November 13-19, 2009, Lake Buena Vista, FL, USA.

[18] K. Nithyanandam, R. Pitchumani, A. Mathur, Analysis of latent thermocline storage system with encapsulated phase change materials for concentrating solar power, Applied Energy 113 (2014) 1446-1460.

[19] S. M. Flueckiger, S. V. Garimella, Latent heat augmentation of thermocline energy storage for concentrating solar power - A system-level assessment. Applied Energy 116 (2014) 278-287.

[20] F. P. Incropera, D. P. DeWitt, Introduction to Heat Transfer, Fourth Edition. John Wiley and Sons, Inc., 2002.

[21] D. E. Beasley, J. A. Clark, Transient Response of a Packed Bed for Thermal Energy Storage. International Journal of Heat and Mass Transfer 27.9 (1984) 1659-1669.

[22] P.W. Li, J. Van Lew, W. Karaki, C.L. Chan, J. Stephens, J.E. O’Brien, Transient heat transfer and energy transport in packed bed thermal storage systems. In book "Developments in Heat Transfer", edited by Marco Aurélio dos Santos Bernardes, ISBN 978-953-307-569-3, 2011.

[23] G. Nellis, S. Klein, Heat Transfer, Cambridge University Press, 2009. 
[24] E. Tumilowicz, C.L. Chan, B. Xu, P.W. Li, An Enthalpy Formulation for Thermocline with Encapsulated PCM Thermal Storage and Benchmark Solution Using the Method of Characteristics, Proceedings of ASME 2013 Summer Heat Transfer Conference, July 14-18, 2013, Minneapolis, MN, USA.

[25] B. Xu, P.W. Li, C.L. Chan, Extending the validity of lumped capacitance method for large Biot number in thermal storage application. Solar Energy, 86.6 (2012) 1709-1724.

[26] P.W. Li, B. Xu, J.X. Han, Y.P. Yang, Verification of a model of thermal storage incorporated with an extended lumped capacitance method for various solid-fluid structural combinations. Solar Energy, 105 (2014) 71-81.

[27] J. H. Ferziger, Numerical Methods for Engineering Applications. Vol. 1, New York: Wiley, 1998. 